\title{
High-Performance Liquid Chromatography Quadrupole Time-of-Flight Mass Spectrometry (HPLC-QTOFMS) Analysis on the Ethanol:Water (80:20) Extract of Lawsonia inermis Leaves \\ (Analisis Cecair Kromatografi Berprestasi Tinggi Kuadrupol Spektrometri Jisim Masa Penerbangan (HPLC-QTOFMS) pada Etanol:Air (80:20) Ekstrak daun Lawsonia inermis)
}

\author{
Muhammad Remy Othman*, Rozana Othman, AbDul Aziz Ismail, HazRina Hazni, KhadHer Ahmad, \\ MuniRAH ABD RAZZAK, ZulKifli MoHd Yusoff \& KHALiJAH AWANG
}

\begin{abstract}
Lawsonia inermis (Henna), (Lythraceae), contains a high amount of phenolic compounds which could activate antioxidants to help reduce free radicals. In this study, the compound content found in the ethanol:water (80:20) extract of local Lawsonia inermis was determined using HPLC-QTOFMS. The ${ }^{1} H$-NMR results were used to determine the peak that exists for the group compounds. Chromatographic peaks were detected and integrated by the MassHunter Acquisition B.07.00 for the Agilent TOF and QTOF and MassHunter Qualitative Analysis B.07.00. The ethanol:water (80:20) extract of L. inermis have shown, predominantly, the presence of phenolic compounds (coumarins, flavonoids, naphthalene, and gallic acid) which are highly glycosylated. The presence of compounds such as apiin, lawsone, apigenin, luteolin, cosmosiin, and p-coumaric acid were also found.
\end{abstract}

Keywords: Ethanol:water (80:20); ${ }^{1} H-N M R$; HPLC-QTOFMS; leaves Lawsonia inermis Malaysia

\section{ABSTRAK}

Lawsonia inermis (inai), (Lythraceae), mengandungi sejumlah besar sebatian fenolik yang boleh mengaktifkan antioksidan dalam membantu mengurangkan radikal bebas. Dalam kajian ini, kandungan sebatian yang terdapat dalam etanol:air (80:20) ekstrak Lawsonia inermis tempatan ditentukan menggunakan HPLC-QTOFMS. Hasil ${ }^{1} H$-NMR digunakan untuk menentukan puncak kumpulan sebatian. Puncak kromatografi dikesan dan diintegrasikan oleh MassHunter Acquisition B.07.00 untuk Agilent TOF dan QTOF dan Analisis Kualitatif MassHunter B.07.00. Daripada etanol:air (80:20) ekstrak L. inermis menunjukkan sebahagian besarnya, kehadiran sebatian fenolik (kumarin, flavonoid, naftalena dan asid galik) yang sangat glikosilasi. Kehadiran sebatian seperti apiin, lawson, apigenin, luteolin, kosmosiin dan asid-p-kumarit turut dijumpai.

Kata kunci: Daun Lawsonia inermis Malaysia; etanol:air (80:20); ${ }^{1} H-N M R$; HPLC-QTOFMS

\section{INTRODUCTION}

Lawsonia inermis (family Lythraceae) is a plant that has been widely studied and used throughout the world for traditional medicinal and cosmetic. Globally, Lawsonia inermis is used as a traditional or folk medicine for the treatment of a wide range of seemingly unrelated ailments such as induce abortion (Africa) diuretic, gonorrhoea and bronchitis (Cambodia), pain and skin affections intestinal amoebiasis (Egypt), headache, burning of skin, decoction used for sore throat (Ahmad \& Beg 2001) jaundice and other liver disorders, itching and other skin disorders (India, Pakistan), hair tonic (India, Jordan), fever, malaria, as a blood tonic (Nigeria), boils, conjunctivitis, pimples, dandruff, scabies, baldness and other scalp disorders (Rusia, Asia) (Semwal et al. 2014).
In Malaysia, people call it 'Inai' and it is used as coloring fingernails especially in weddings but not extensively used in medicine such as fungal pathogens treatment, head lice treatment, reduce dandruff, and also for digestive disorders, treating diabetics and ulcers (Othman et al. 2020).

Lawsonia inermis contain high amount of phenolic compounds which activate antioxidants to help reduce free radicals (Oroian \& Escriche 2015). Many studies on $L$. inermis have found nearly 70 phenolic compounds that have been isolated from various parts of $L$. inermis include root, bark, flower, and leaves where it is proven to have various active compounds (Semwal et al. 2014). Lawsone (2-hydroxy-1, 4 naphthaquinone) is a natural pigment present in the leaves of $L$. inermis. It is the principal active ingredient of the henna plant 
(Saeed 2013). Lawsone, also known as hennotannic acid chemically, lawsone is similar to juglone, which is found in walnuts (Babula et al. 2005; Ebrahimi \& Parvinzadeh Gashti 2016).

Throughout the world, proactive studies have proven scientifically the effectiveness of inorganic $L$. inermis pharmacological activities such as antibacterial (Jabborova et al. 2019), virucidal, antiparasitic, antiinflammatory, analgesic, and anticancer properties, as well as hepatoprotective, immunomodulatory, anthelminthic, antitrypanosomal, and antioxidant activities (Chaudhary et al. 2010; Pasandi Pour \& Farahbakhsh 2019; Semwal et al. 2014). For compounds identification, we analysis with high-performance liquid chromatography (HPLC) (Acquaviva et al. 2018). Then, ${ }^{1} \mathrm{H}-\mathrm{NMR}$ result produced was used to determine the peak that exists for the group compounds (Chandrakalavathi et al. 2018). The objective of this study was to analyze the compounds content found in the local $L$. inermis using ethanol-water mixtures. This is the most suitable solvent system for the extraction of $L$. inermis due to the different polarities of the active constituents, and the acceptability of the solvent system for human consumption (Alothman 2009; Gull et al. 2013) even though the best solution was acetone followed by ethanol (Tan 2013).

\section{MATERIALS AND METHODS}

\section{SAMPLE COLLECTION}

Lawsonia inermis leaves were collected from the residential area of Sentosa, Bandar Baru Bangi, Selangor,
Malaysia. The collection was led by Mr. Din Bin Md Nor and the botanical identification was made by Mr. Teoh Leong Eng from the Department of the Chemistry University of Malaya. A voucher specimen KL5824 was deposited to the herbarium of Chemistry, Faculty of Science, University of Malaya.

\section{PREPARATION OF THE EXTRACTS}

Dried and ground of Lawsonia inermis leaves (300 g) was soaked in $80 \%$ ethanol and $20 \%$ distilled water. The sample was macerated under room temperature for three consecutive days. Then, the suspension was filtered using filter paper. The eluent was first concentrated using rotary evaporator to remove the ethanol solvent followed with freeze drying method to remove the distilled water to give water extracts (LILEW). About $1.2 \mathrm{~g}$ of LILEW were dissolved in distilled water before mix with dichloromethane to form an immiscible solution. The two phases were agitated, by shaking and swirl to bring about substantial physical mixing. After agitation, the phases were allowed to separate. Compounds having different solubilities will be separated base on the polarity of the solvent. Sodium sulfate anhydrous $\left(\mathrm{Na}_{2} \mathrm{SO}_{4}\right)$ were use in the organic layer as a drying agent. The dichloromethane solution was concentrated to dryness using rotary evaporator and kept in the fridge until further used (DCM extract). The aqueous solution was then mix with ethyl acetate and liquid-liquid partition were repeated to give EA extract. The LILEW, DCM and EA extracts were weight and recorded (Table 1) and analyze using ${ }^{1} \mathrm{H}-\mathrm{NMR}$ as shown in Figures 1 to 3.

TABLE 1. The yield of DCM partition and EA partition for aqueous extraction LILEW

\begin{tabular}{ccc}
\hline No. & Extract & Remaining yield \\
\hline 1 & LILEW & $36 \mathrm{~g}$ \\
2 & DCM & $97 \mathrm{mg}$ \\
3 & EA & $156 \mathrm{mg}$ \\
\hline
\end{tabular}

\section{NUCLEAR MAGNETIC RESONANCE SPECTROSCOPY (NMR)}

One-dimensional ${ }^{1} \mathrm{H}$-NMR experiments were carried out on JEOL ECA 400 FT-NMR. $\mathrm{CDCl}_{3}$ and MeOD were used as the solvent depending on the polarity. The chemical shifts were recorded with reference to $\mathrm{CDCl}_{3}(7.24 \mathrm{ppm})$ or MeOD (4.78 ppm).

LC-MS chromatography were carried out on Agilent 1260 Infinity series ultra-high-performance liquid chromatography (UHPLC) system and a G6530B accurate- mass Q-TOF LC/MS instrument (Agilent Technologies, Santa Clara, USA). $10 \mu \mathrm{L}$ of extracts were injected and chromatographed on a ZORBAX Eclipse Plus C8 column $(100 \times 2.1 \mathrm{~mm}$ id, $1.8 \mu \mathrm{m})$ column with the mobile phase of A ( $0.1 \%$ formic acid in water) and B (methanol). The mobile phase was performed as follows: Gradient from 5 to $100 \%$ B over the next $30 \mathrm{~min}$, isocratic at $100 \% \mathrm{~B}$ for $5 \mathrm{~min}$ and re-equilibrated for $5 \mathrm{~min}$ at $5 \% \mathrm{~B}$ before the next run. Eluent were monitored using diode array 
detector at 220, 256, and $380 \mathrm{~nm}$. ESI-MS was performed in the range 100 to 1,000 in both the positive and negative mode. The temperature was set to $300{ }^{\circ} \mathrm{C}$. Nitrogen gas was used as nebulizer with the flow rate set at $10 \mathrm{~L} \mathrm{~min}^{-1}$. The ESI source voltage was set at $3.5 \mathrm{kV}$. Mass Hunter Acquisition B.07.00 software was used for data analysis.

\section{RESULTS AND DISCUSSION}

\section{NUCLEAR MAGNETIC RESONANCE SPECTROSCOPY (NMR)}

${ }^{1} \mathrm{H}-\mathrm{NMR}$ studies are presented in Figures 1 to 3. In Figure 1, ${ }^{1} \mathrm{H}-\mathrm{NMR} 400 \mathrm{MHz}$ of aqueous extraction of LILEW, the result shows the chemical shifts of the aromatic group (6.5-8.5 ppm), methoxyl group $\left(\mathrm{CH}_{2} \mathrm{OH} / \mathrm{CH}-\mathrm{OH}\right)\left(\mathrm{CH}_{2} \mathrm{O} /\right.$ $\mathrm{CH}-\mathrm{O}$ ) in the range 3.0 to $4.5 \mathrm{ppm}$. In Figure 2, ${ }^{1} \mathrm{H}-\mathrm{NMR}$ $400 \mathrm{MHz}$ of $L$. inermis extract result for DCM partition the chemical shift of the methyl $\left(\mathrm{CH}_{3}\right)$, methylene $\left(\mathrm{CH}_{2}\right)$, methane $(\mathrm{CH})$ between 0.5 and $2.0 \mathrm{ppm}$ similar with aqueous extraction of LILEW. Figure 3 shows ${ }^{1} \mathrm{H}-\mathrm{NMR}$
$400 \mathrm{MHz}$ of L. inermis EtOH:water extract result for EA partition show the best and clear chemical shifts of the aromatic group, between 6.5 and $8.5 \mathrm{ppm}$ compare with DCM partition. We decide to run LC-MS profile using EA partition for future investigation of potential compounds from aqueous extraction of LILEW.

\section{LIQUID CHROMATOGRAPHY-MASS SPECTROMETRY (LC-MS) PROFILE ANALYSIS}

The LC-MS chromatogram was generated after analysis of the reference standards using the UHPLC-QTOFMS method as described above. Figure 4 shows LC-MS peak/ total ion chromatogram (TIC) for LILEW EA partition (negative mode) where Figure 5 shows LC-MS peak number of selected compounds for LILEW extract using EA partition. From the result of LC-MS profile, we manage to identify one major class of compound from phenolic. We have chosen 27 peak number of potential compounds.

TABLE 2. List of compounds, chemical formula, exact mass, [M-H]- and retention time

\begin{tabular}{|c|c|c|c|c|c|c|}
\hline $\begin{array}{l}\text { Peak } \\
\text { label }\end{array}$ & Major classes & Compound name & $\begin{array}{l}\text { Chemical } \\
\text { formula }\end{array}$ & Exact mass & $\begin{array}{l}\text { Mass detected } \\
\text { under negative } \\
\text { mode }[\mathrm{M}-\mathrm{H}]-\end{array}$ & $\begin{array}{l}\text { Retention time } \\
\left(\mathrm{R}_{\mathrm{t}}\right) / \min \end{array}$ \\
\hline 1 & Coumarins & Scopoletin & $\mathrm{C}_{10} \mathrm{H}_{8} 0_{4}$ & 192.0423 & 191.0208 & 1.155 \\
\hline 2 & Tannins & $\begin{array}{c}\text { 1,2,3,6-Tetra-O-glalloyl- } \\
\text { B-D-Glocose }\end{array}$ & $\mathrm{C}_{34} \mathrm{H}_{28} \mathrm{O}_{22}$ & 788.1072 & 787.1068 & 1.155 \\
\hline 3 & $\begin{array}{l}\text { Trihydroxybenzoic } \\
\text { acid }\end{array}$ & Gallic Acid & $\mathrm{C}_{7} \mathrm{H}_{6} \mathrm{O}_{5}$ & 170.0215 & 169.0142 & 3.163 \\
\hline 4 & Naphthoquinones & 4-Hydroxy-a-tetralone & $\mathrm{C}_{10} \mathrm{H}_{10} \mathrm{O}_{2}$ & 162.0681 & 161.0461 & 5.586 \\
\hline 5 & Flavonoids & $\begin{array}{l}\text { 2,4-Dihydroxybenzoic } \\
\text { acid }\end{array}$ & $\mathrm{C}_{7} \mathrm{H}_{6} \mathrm{O}_{4}$ & 154.0266 & 153.0193 & 7.843 \\
\hline 6 & Coumarins & $\begin{array}{l}\text { Agrimonolide 6-O- } \beta \text {-D- } \\
\text { glucopyranoside }\end{array}$ & $\mathrm{C}_{24} \mathrm{H}_{28} \mathrm{O}_{10}$ & 476.1682 & 475.0885 & 7.926 \\
\hline 7 & Flavonoids & Acacetin-7-O-glucoside & $\mathrm{C}_{22} \mathrm{H}_{22} \mathrm{O}_{10}$ & 446.1213 & 445.0859 & 8.623 \\
\hline 8 & Flavonoids & Lawsochrysinin & $\mathrm{C}_{20} \mathrm{H}_{18} \mathrm{O}_{4}$ & 322.1205 & 321.0615 & 8.955 \\
\hline 9 & Coumarins & Esculetin & $\mathrm{C}_{9} \mathrm{H}_{6} \mathrm{O}_{4}$ & 178.0266 & 177.0194 & 9.171 \\
\hline 10 & Coumarins & Fraxetin & $\mathrm{C}_{10} \mathrm{H}_{8} \mathrm{O}_{5}$ & 208.0372 & 207.0307 & 9.436 \\
\hline 11 & Alkylphenones & Lalioside & $\mathrm{C}_{14} \mathrm{H}_{18} 0_{14}$ & 346.09 & 345.0835 & 11.594 \\
\hline 12 & Flavonoids & 3',4' dimethoxyflavone & $\mathrm{C}_{17} \mathrm{H}_{14} \mathrm{O}_{4}$ & 282.0892 & 281.2482 & 11.992 \\
\hline 13 & Flavonoids & Lawsochrysin & $\mathrm{C}_{25} \mathrm{H}_{30} \mathrm{O}_{4}$ & 394.2144 & 393.0457 & 12.208 \\
\hline 14 & Tannins & $\begin{array}{c}\text { 1,2,3,4,6-penta-O-galloly- } \\
\text { B-D-glucose }\end{array}$ & $\mathrm{C}_{41} \mathrm{H}_{32} \mathrm{O}_{26}$ & 940.1182 & 939.1144 & 12.988 \\
\hline 15 & Flavonoids & Luteolin-7-o-glucoside & $\mathrm{C}_{21} \mathrm{H}_{20} \mathrm{O}_{11}$ & 448.1006 & 447.0929 & 14.366 \\
\hline 16 & Flavonoids & Apiin & $\mathrm{C}_{26} \mathrm{H}_{28} \mathrm{O}_{14}$ & 564.1479 & 563.0951 & 14.432 \\
\hline
\end{tabular}




\begin{tabular}{lcccccc}
17 & Naphthoquinones & Lawsone & $\mathrm{C}_{10} \mathrm{H}_{6} \mathrm{O}_{3}$ & 174.0319 & 173.0242 & 14.598 \\
18 & Naphthoquinones & Isoplumbagin & $\mathrm{C}_{11} \mathrm{H}_{8} \mathrm{O}_{3}$ & 188.0473 & 187.0395 & 14.847 \\
19 & Coumarins & Daphnorin & $\mathrm{C}_{25} \mathrm{H}_{22} \mathrm{O}_{12}$ & 514.1111 & 513.1401 & 15.411 \\
20 & Flavonoids & $\begin{array}{c}\text { Cosmosin/Apigenin-7-O- } \\
\text { B-D-glucopyranoside }\end{array}$ & $\mathrm{C}_{21} \mathrm{H}_{20} \mathrm{O}_{10}$ & 432.1056 & 413.1001 & 15.610 \\
21 & Flavonoids & Scutellarin & $\mathrm{C}_{21} \mathrm{H}_{18} \mathrm{O}_{12}$ & 462.0798 & 461.1117 & 16.407 \\
22 & Flavonoids & Luteolin & $\mathrm{C}_{15} \mathrm{H}_{10} \mathrm{O}_{6}$ & 286.0477 & 285.0408 & 18.631 \\
23 & Flavonoids & Apigenin & $\mathrm{C}_{15} \mathrm{H}_{10} \mathrm{O}_{5}$ & 270.0528 & 269.0461 & 20.025 \\
24 & Coumarins & Daphneside & $\mathrm{C}_{21} \mathrm{H}_{26} \mathrm{O}_{14}$ & 502.1323 & 501.1062 & 22.763 \\
25 & Flavonoids & Acacetin & $\mathrm{C}_{16} \mathrm{H}_{12} \mathrm{O}_{5}$ & 284.0685 & 283.1391 & 25.004 \\
26 & Flavonoids & $3,, 4$ ' dimethoxy flavone & $\mathrm{C}_{17} \mathrm{H}_{14} \mathrm{O}_{4}$ & 298.0841 & 297.1536 & 25.651 \\
27 & Coumarins & Lacoumarin & $\mathrm{C}_{12} \mathrm{H}_{10} \mathrm{O}_{4}$ & 218.0579 & 217.0489 & 38.198 \\
\hline
\end{tabular}

This work describes a comprehensive and inclusive technique for the characterization of major constituents for the ethanol:water (80:20) extract of the leaves of the Malaysian L. inermis. Twenty-seven compounds were identified to be phenolic compounds with exact mass, molecular weight, chemical formula, and retention time.

This paper also provides information on nuclear magnetic resonance spectroscopy (NMR) and followed by high-performance liquid chromatography (HPLC) methods to make comprehensive studies. This finding also suggested the mixtures of $80 \%$ ethanol with $20 \%$ water suitable solvent system for the extraction of $L$. inermis due to the different polarities of the active constituents, and the acceptability of the solvent system for human consumption (Alothman 2009).

Peak no. 1 shows compound scopoletin type of coumarin (Figure 6) (Agarwal et al. 2014). Figure 7 shows peak no. 2 for compound 1,2,3,6-tetra-O-glalloylB-D-glucose might be an anti-hepatitis B virus (HBV) agent (Xiang et al. 2010). Figure 8 shows peak no. 3 for compound gallic acid. Gallic acid has been reported to have therapeutic activities in gastrointestinal, antioxidant, and anti-inflammatory (Kahkeshani 2019). In Figure 9, peak no. 4 indicated compound 4-hydroxy-a-tetralone and peak no. 5 shows the fragmentation pattern of compound 2,4-dihydroxybenzoic acid (Figure 10) of the major peak with $\mathrm{m} / \mathrm{z}$ value 153.0193 .

Peak no. 6 indicated of compound agrimonolide 6-O- $\beta$-D-glucopyranoside shown in Figures 11 and 12 show peak no. 7 with $\mathrm{m} / \mathrm{z}$ value 445.0859 indicate mass detected under negative mode $[\mathrm{M}-\mathrm{H}]-$ of compound acacetin-7-O-glucoside (Muhammad \& Muhammad 2005; Sharma et al. 2016). Figure 13 indicated the compound name lawsochrysinin (peak no. 8) (Uddin et al. 2013).

Figure 18 shows compound lawsochrysin (peak no. 13) (Brahmachari 2018). Figure 19 shows compound name 1,2,3,4,6-penta-O-galloly- $\beta$-D-glucose indicated in peak no. 14. Peak no. 19 for compound daphnorin has shown in Figure 24. We also manage to identify compound daphneside (peak no. 24) (Figure 29) and for peak no. 27 indicated compound lacoumarin (Figure 32).

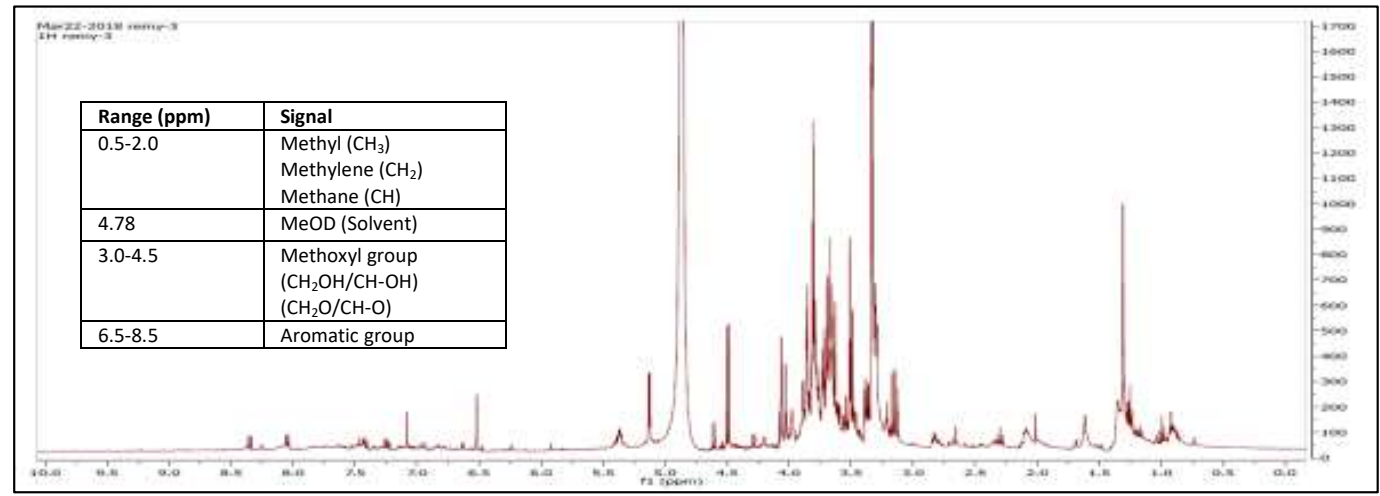

FIGURE 1. ${ }^{1} \mathrm{H}-\mathrm{NMR} 400 \mathrm{MHz}$ of aqueous extraction of LILEW 


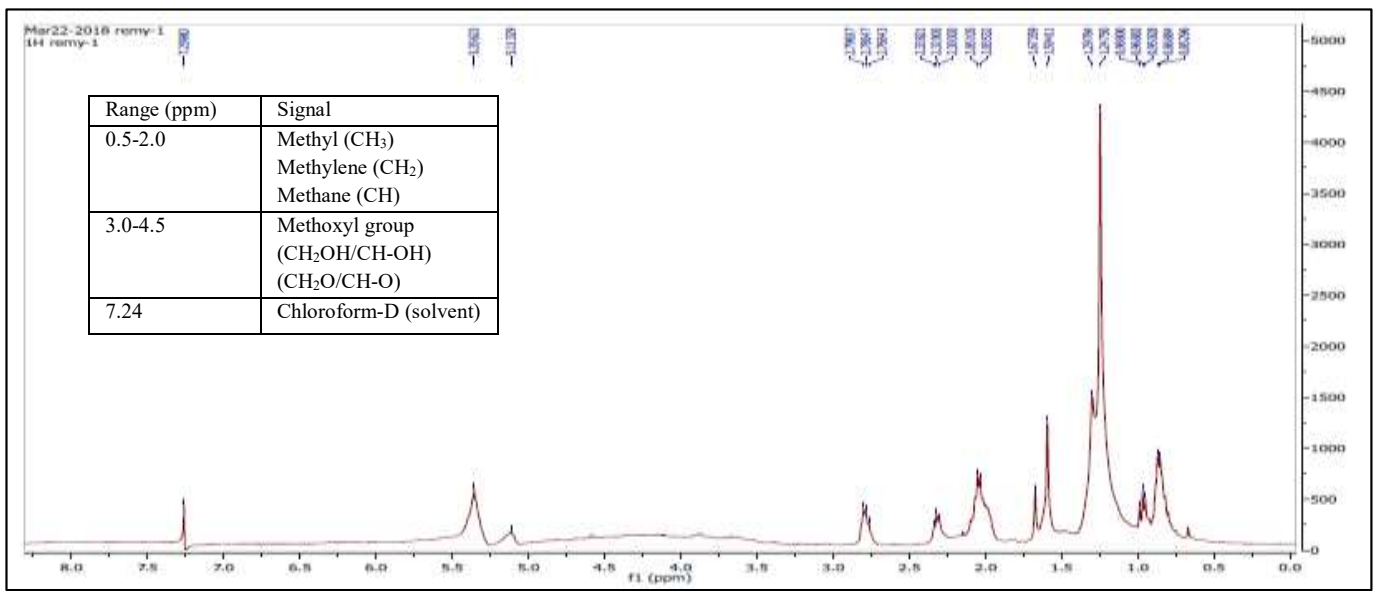

FIGURE 2. ${ }^{1} \mathrm{H}-\mathrm{NMR} 400 \mathrm{MHz}$ of LILEW extract result for DCM Partition

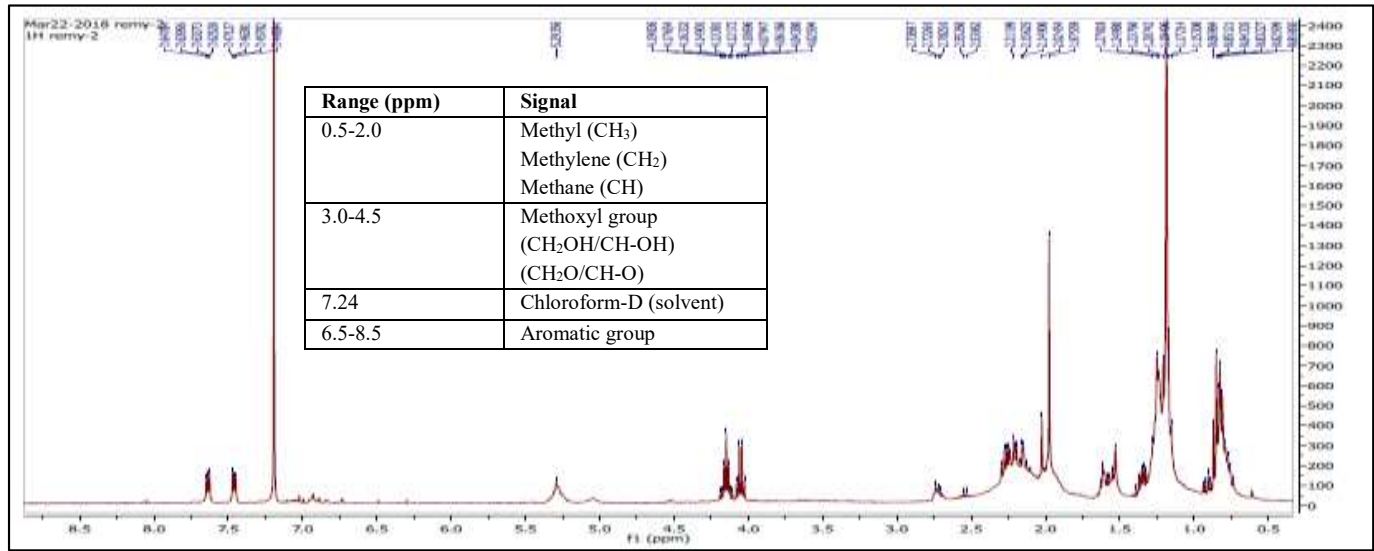

FIGURE 3. ${ }^{1} \mathrm{H}-\mathrm{NMR} 400 \mathrm{MHz}$ of LILEW extract result for EA Partition

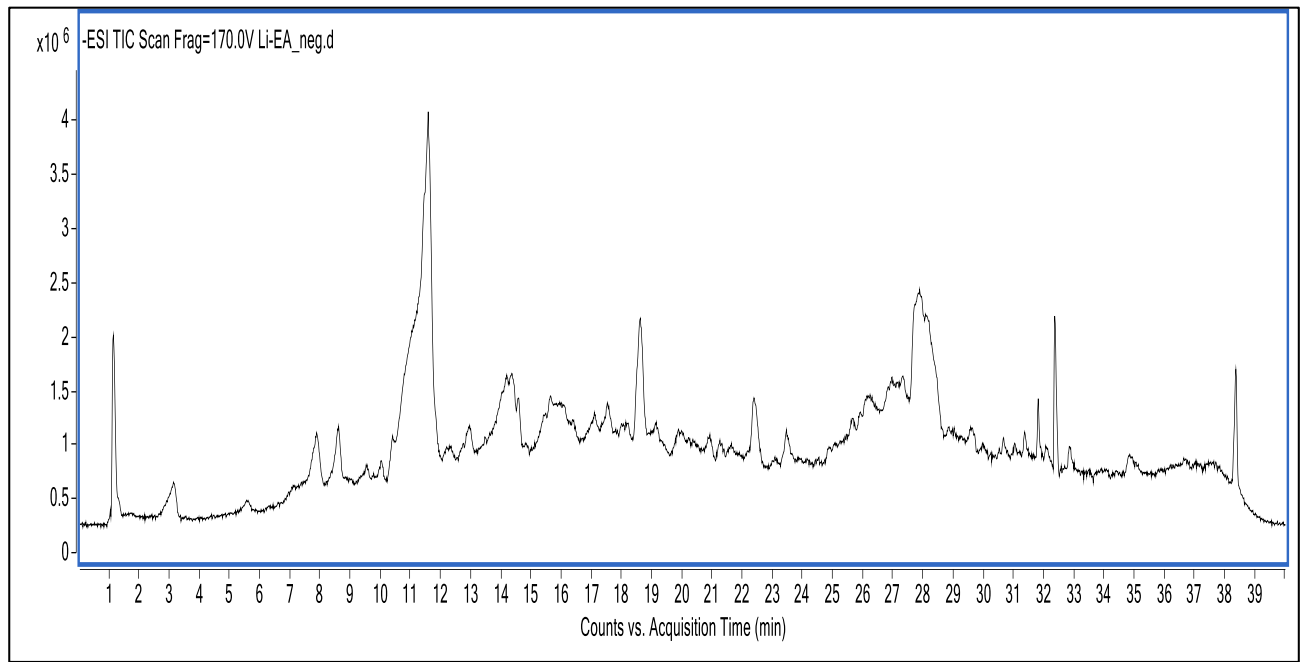

FIGURE 4. LC-MS Peak/Total Ion Chromatogram (TIC) for Lawsonia inermis EtOH: Water of EA partition (Negative mode 


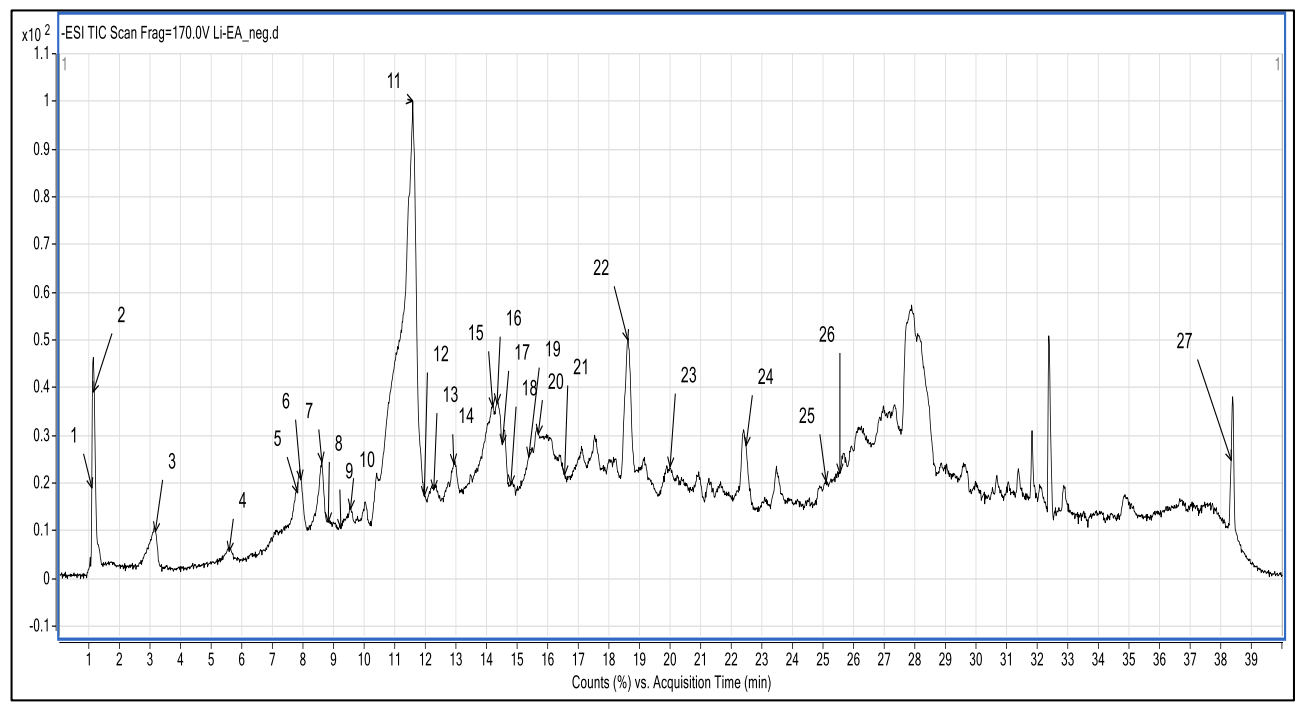

FIGURE 5. LC-MS Peak number of Compounds for EA Partition of aqueous LILEW extract in Negative mode

Peak no. 1, Name: Scopoletin

Exact Mass: $192.0423,(\mathrm{~m} / \mathrm{z}-\mathrm{H}) ;[\mathrm{m} / \mathrm{z}-1]=191.0432$ (RT: $1.1155 \mathrm{~min})$
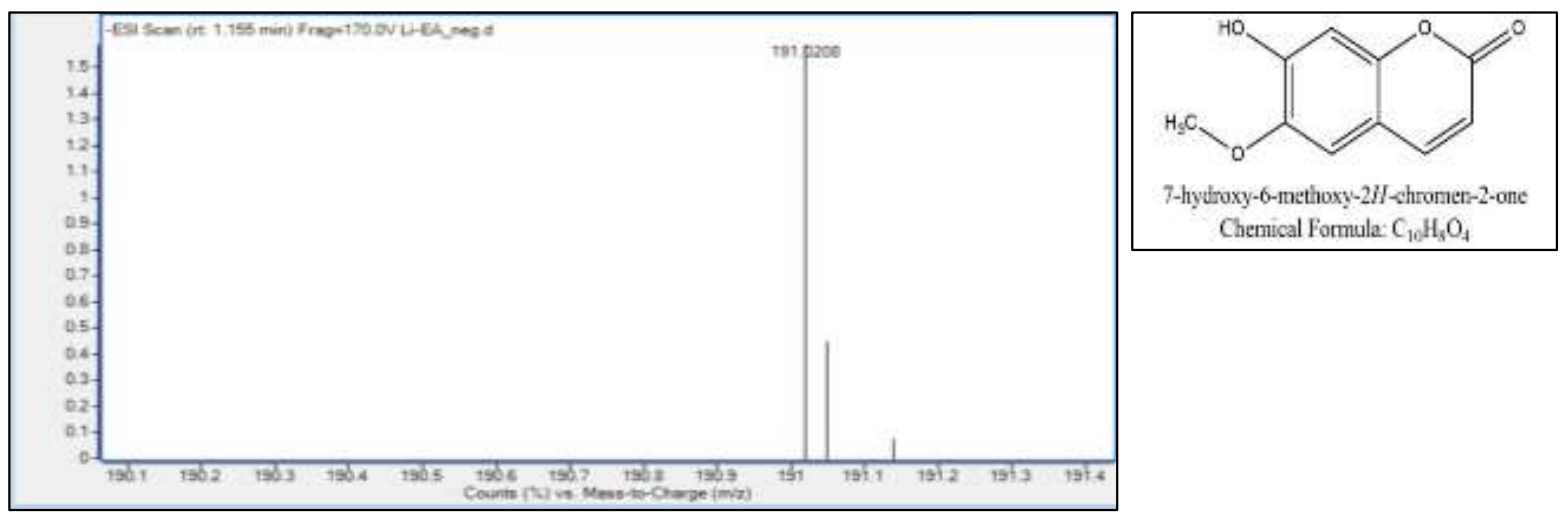

FIGURE 6. Fragmentation pattern from MS/MS spectrum of the major peak label no. 1 with $\mathrm{m} / \mathrm{z}$ value 191.0208

Peak no. 2, Name: 1,2,3,6-Tetra-O-glalloyl-B-D-Glocose

Exact Mass: $788.1072,(\mathrm{~m} / \mathrm{z}-\mathrm{H}) ;[\mathrm{m} / \mathrm{z}-1]=787.1072(\mathrm{RT}: 1.155 \mathrm{~min})$
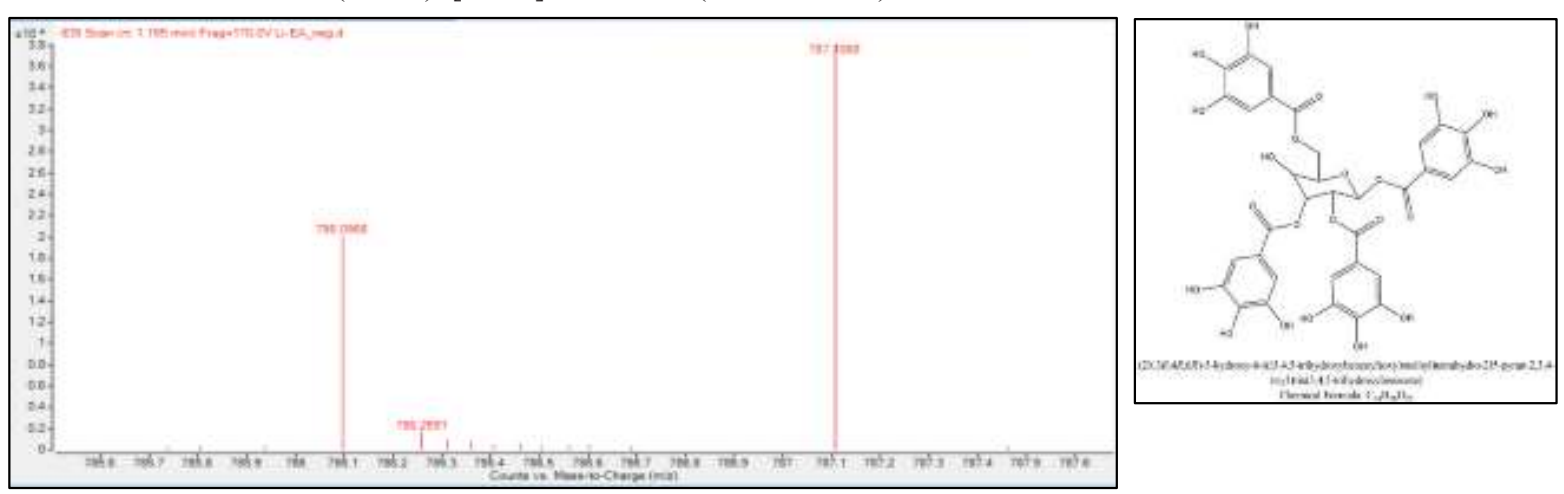

FIGURE 7. Fragmentation pattern from MS/MS spectrum of the major peak with $\mathrm{m} / \mathrm{z}$ value 787.1068 
Peak no. 3, Name: Gallic Acid

Exact Mass: 170.0215, (m/z-H); [m/z-1] $=169.0215$ (RT: $3.163 \mathrm{~min})$

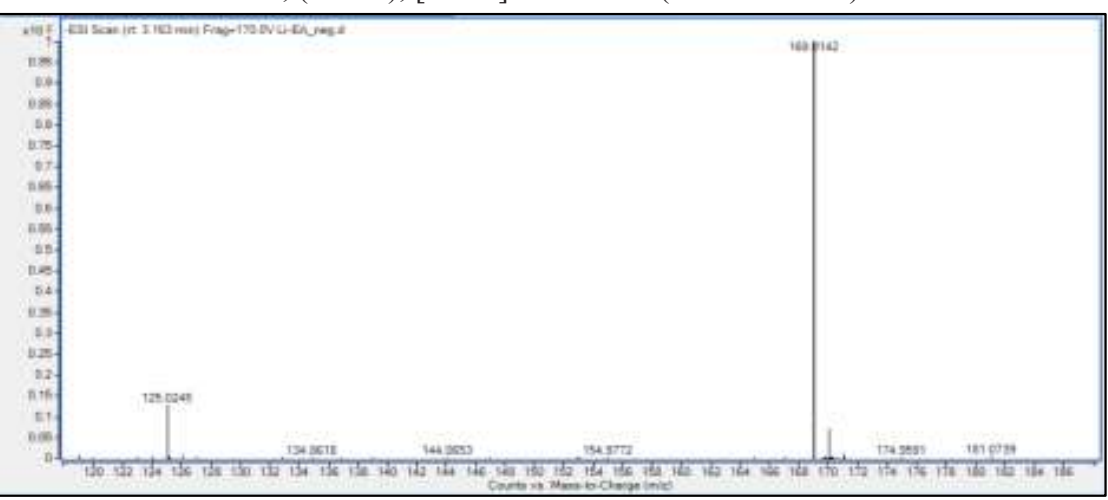

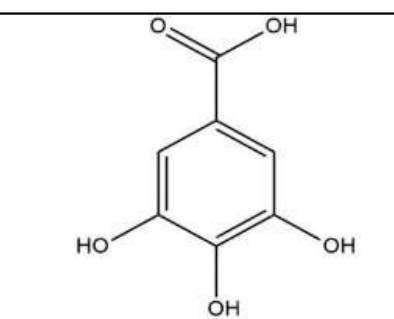

3,4,5-trihydroxybenzoic acid Chemical Formula: $\mathrm{C}_{7} \mathrm{H}_{6} \mathrm{O}_{5}$

FIGURE 8. Fragmentation pattern from MS/MS spectrum of the major peak with $\mathrm{m} / \mathrm{z}$ value 169.0142

Peak no. 4, Name: 4-Hydroxy-a-tetralone.

Exact Mass: 162.0681, (m/z-H); [m/z-1] $=161.0681$ (RT: $5.586 \mathrm{~min})$
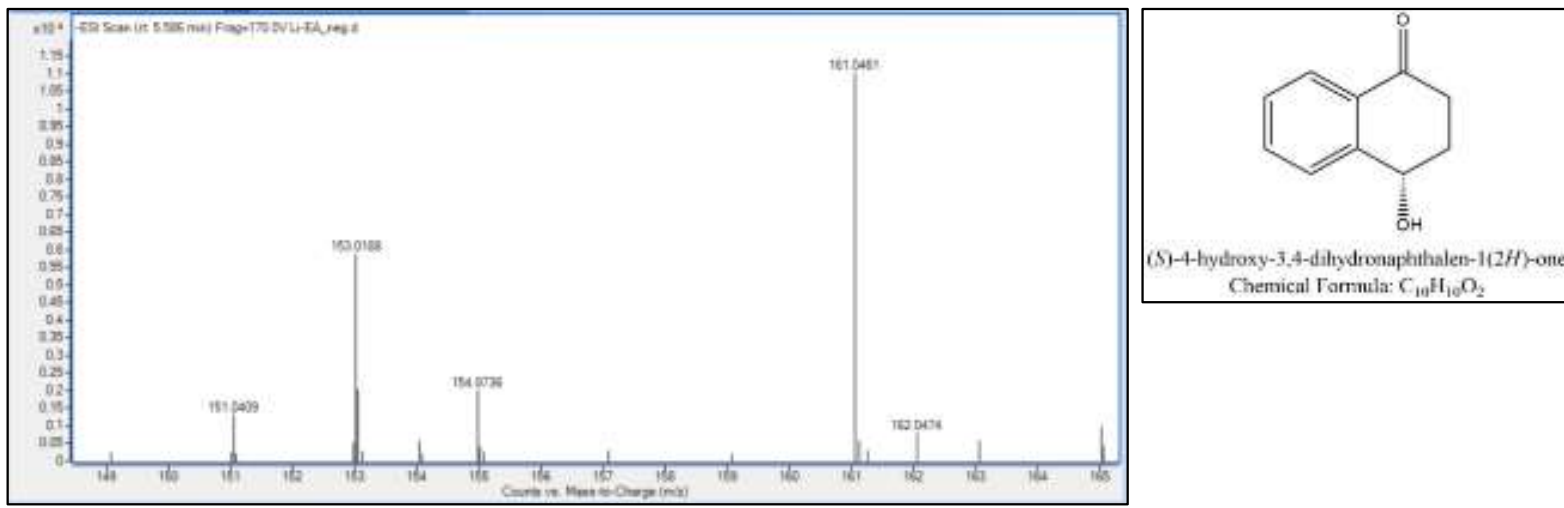

FIGURE 9. Fragmentation pattern from MS/MS spectrum of the major peak with $\mathrm{m} / \mathrm{z}$ value 161.0461

Peak no. 5, Name: 2,4-Dihydroxybenzoic acid

Exact Mass: 154.0266, (m/z-H); [m/z-1] $=153.0266$ (RT: $7.843 \mathrm{~min})$
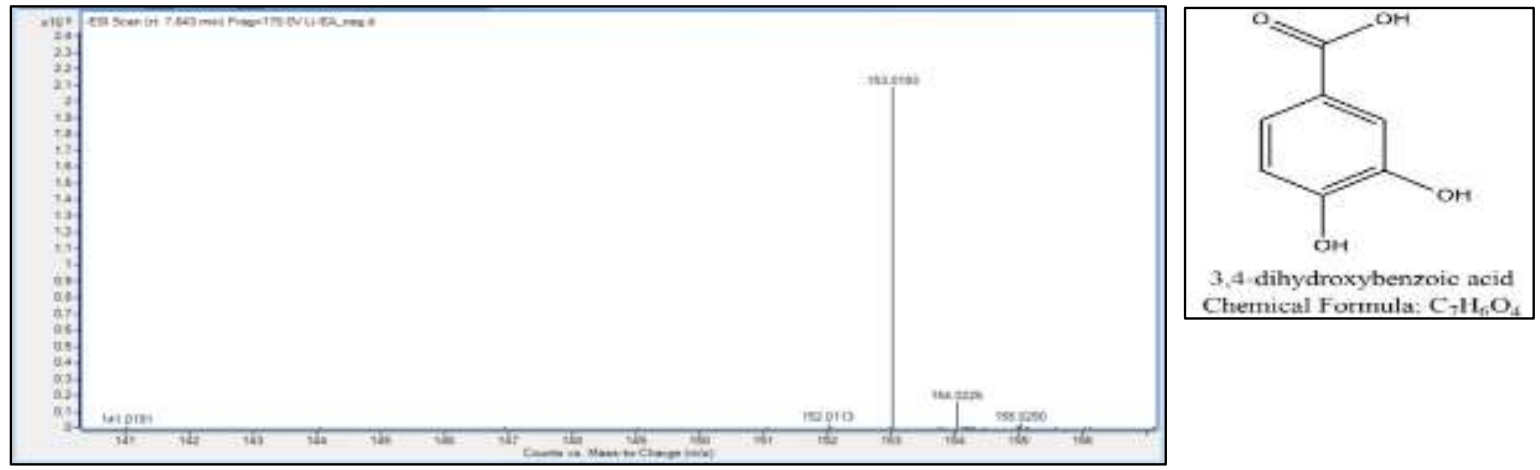

Chemical Formula: $\mathrm{C}_{7} \mathrm{H}_{4} \mathrm{O}_{4}$

FIGURE 10. Fragmentation pattern from MS/MS spectrum of the major peak with $\mathrm{m} / \mathrm{z}$ value 153.0193 
Peak no. 6, Name: Agrimonolide 6-O-B-D-glucopyranoside Exact Mass: 476.1682, (m/z-H); [m/z-1] = 475.1682 (RT: $7.926 \mathrm{~min})$

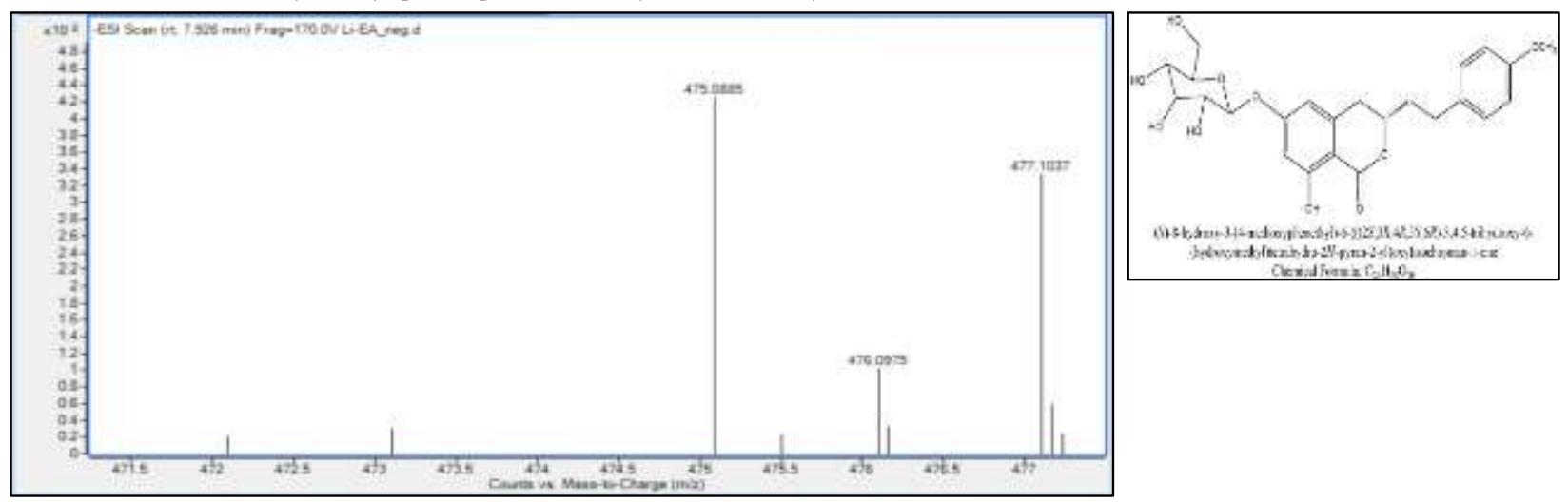

FIGURE 11. Fragmentation pattern from MS/MS spectrum of the major peak with $\mathrm{m} / \mathrm{z}$ value 475.0885

Peak no. 7, Name: Acacetin-7-O-glucoside

Exact Mass: 446.1213, (m/z-H); [m/z-1] $=445.1213$ (RT: $8.623 \mathrm{~min})$
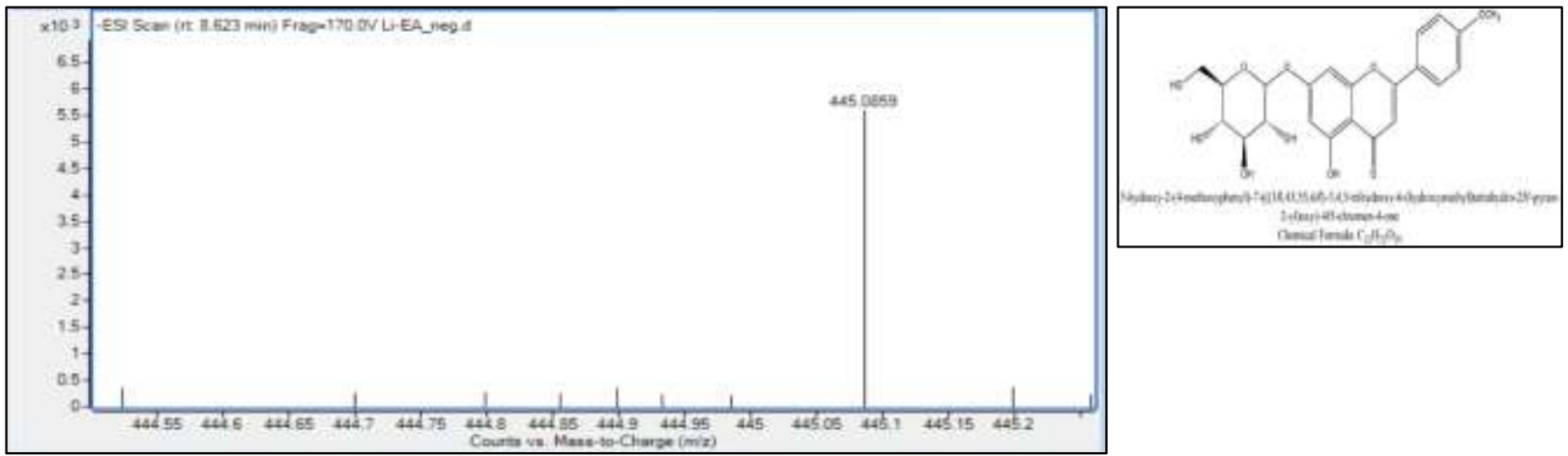

FIGURE 12. Fragmentation pattern from MS/MS spectrum of the major peak with $\mathrm{m} / \mathrm{z}$ value 445.0859

Peak no. 8, Name: Lawsochrysinin

Exact Mass: $322.1205,(\mathrm{~m} / \mathrm{z}-\mathrm{H}) ;[\mathrm{m} / \mathrm{z}-1]=321.1205$ (RT: $8.955 \mathrm{~min})$
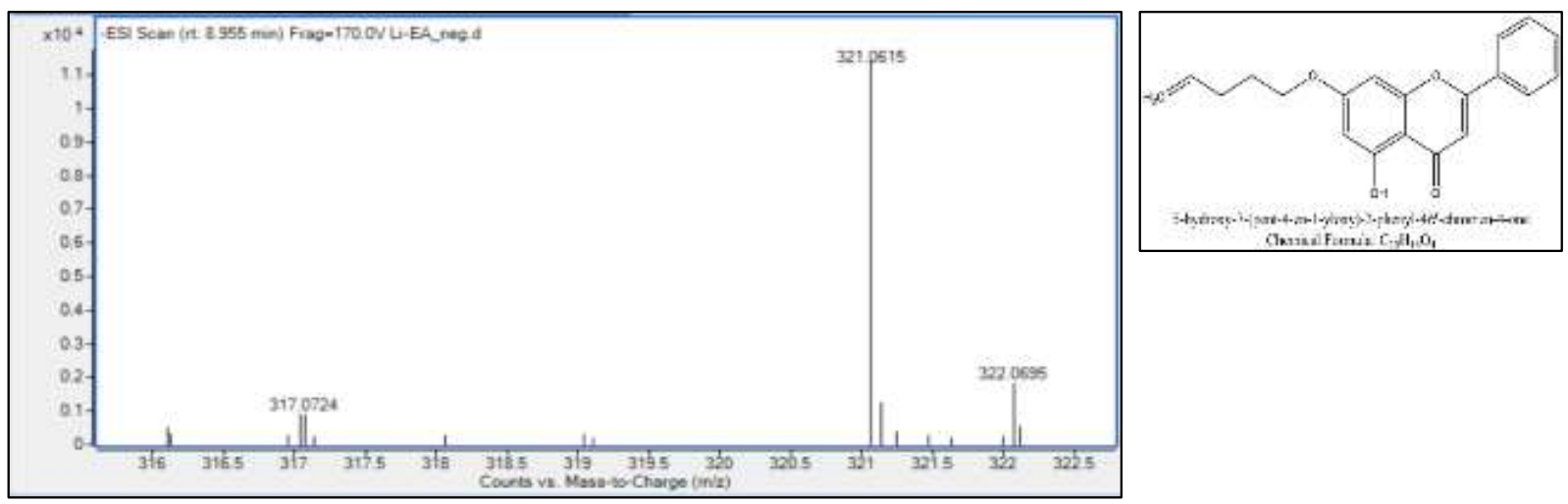

FIGURE 13. Fragmentation pattern from MS/MS spectrum of the major peak with $\mathrm{m} / \mathrm{z}$ value 321.0615 
Peak no. 9, Name: Esculetin

Exact Mass: 178.0266, (m/z-H); [m/z-1] $=177.0266$ (RT: $9.171 \mathrm{~min})$
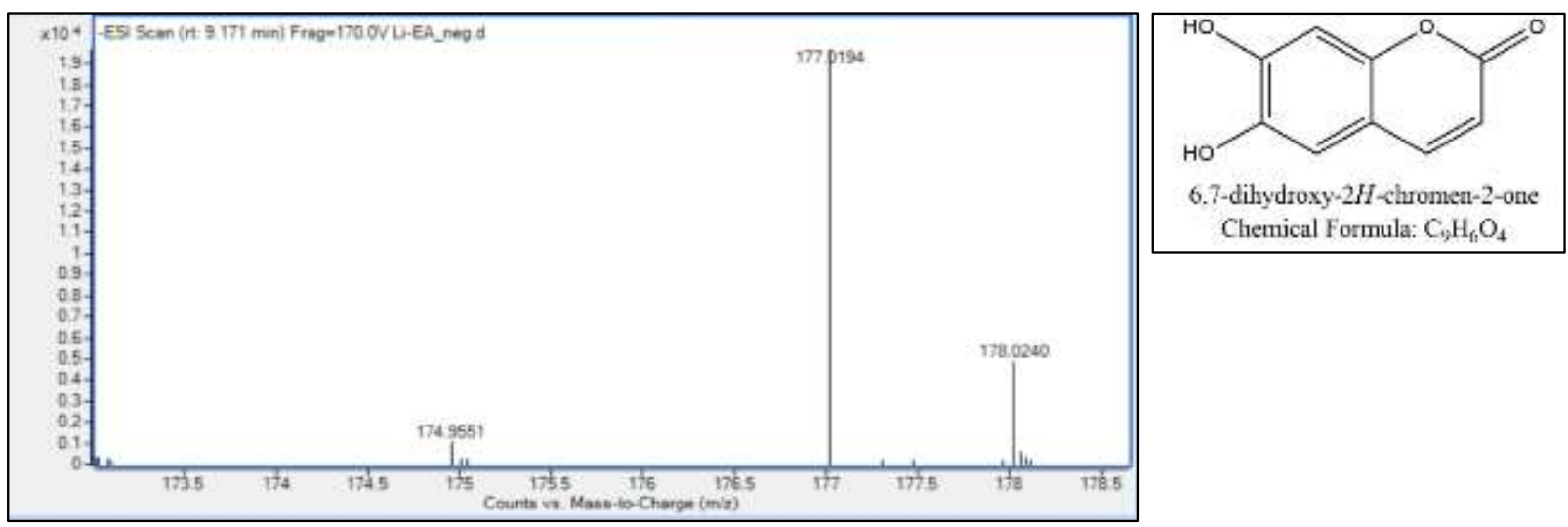

FIGURE 14. Fragmentation pattern from MS/MS spectrum of the major peak with $\mathrm{m} / \mathrm{z}$ value 177.0194

Peak no. 10, Name: Fraxetin

Exact Mass: 208.0372, (m/z-H); [m/z-1] = 207.0372 (RT: 9.436 min)
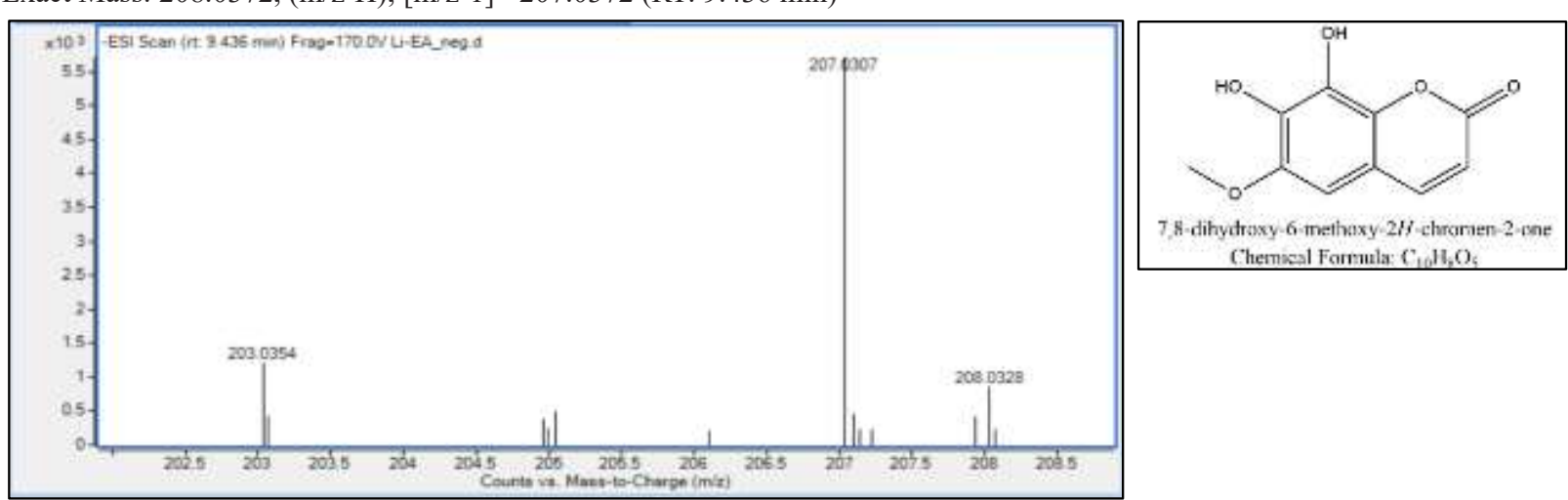

Chemical Formula: $\mathrm{C}_{1} \mathrm{H}, \mathrm{O}$

FIGURE 15. Fragmentation pattern from MS/MS spectrum of the major peak with $\mathrm{m} / \mathrm{z}$ value 207.0307

Peak no. 11, Name: Lalioside

Exact Mass: $346.0900,(\mathrm{~m} / \mathrm{z}-\mathrm{H}) ;[\mathrm{m} / \mathrm{z}-1]=345.0900$ (RT: $11.594 \mathrm{~min})$
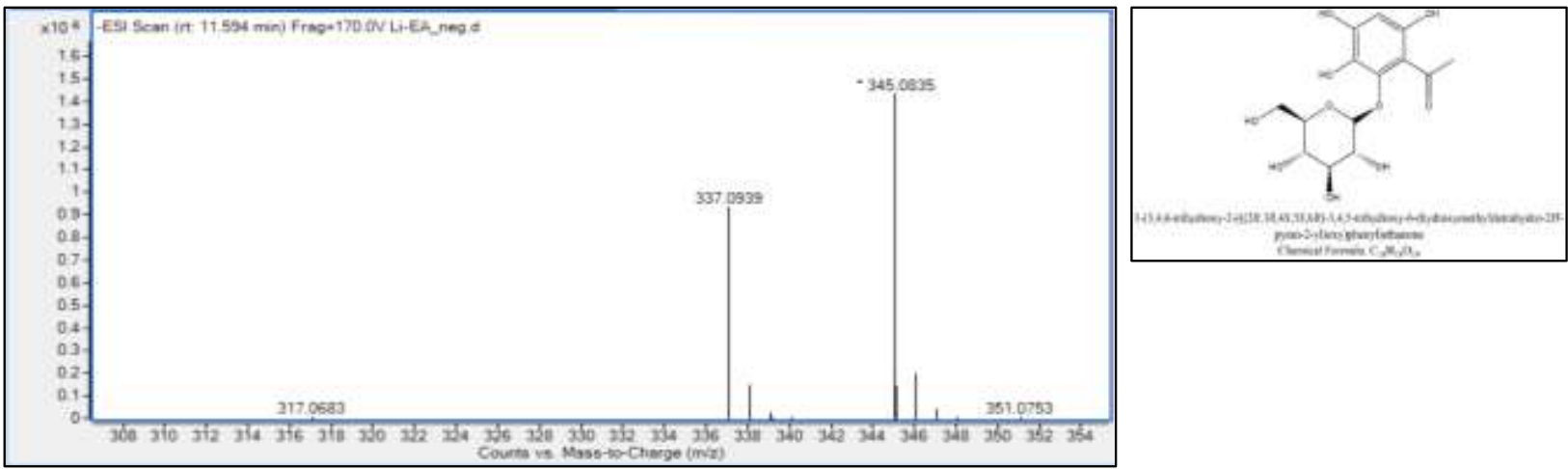

FIGURE 16. Fragmentation pattern from the MS/MS spectrum of the major peak with $\mathrm{m} / \mathrm{z}$ value 345.0835 
Peak no. 12, Name: 3',4' dimethoxyflavone

Exact Mass: 282.0892, (m/z-H); [m/z-1] $=281.0892$ (RT: $11.992 \mathrm{~min})$
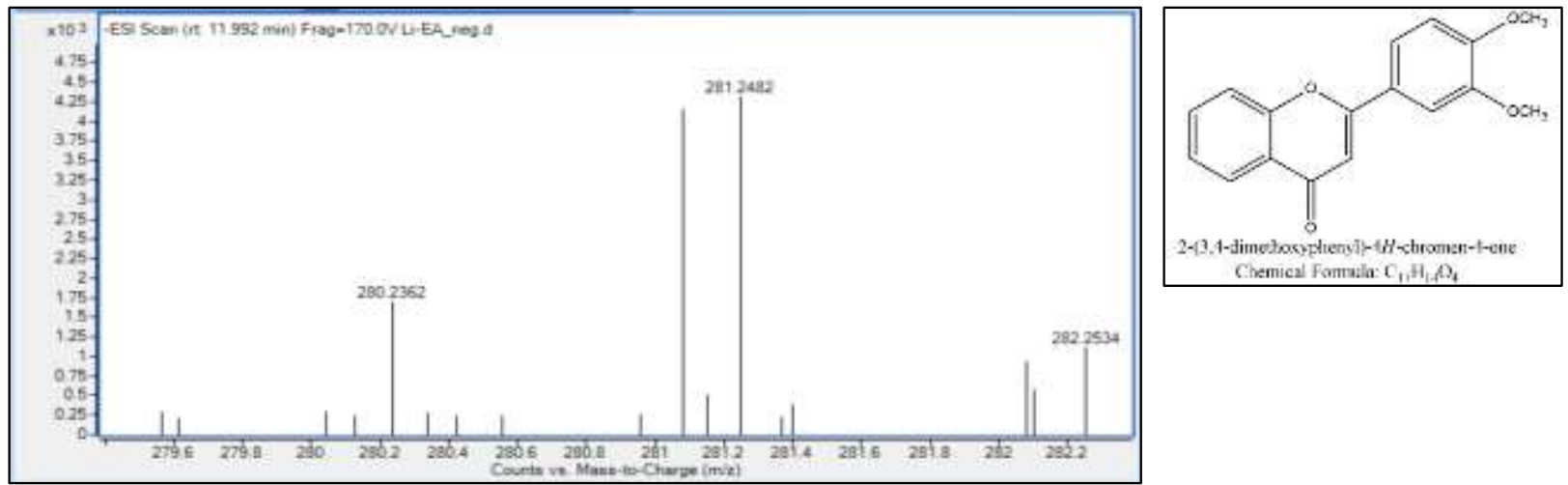

FIGURE 17. Fragmentation pattern from the MS/MS spectrum of the major peak with $\mathrm{m} / \mathrm{z}$ value 281.2482

Peak no. 13, Name: Lawsochrysin

Exact Mass: 394.2144, (m/z-H); [m/z-1] = 393.2144 (RT: 12.208 min)
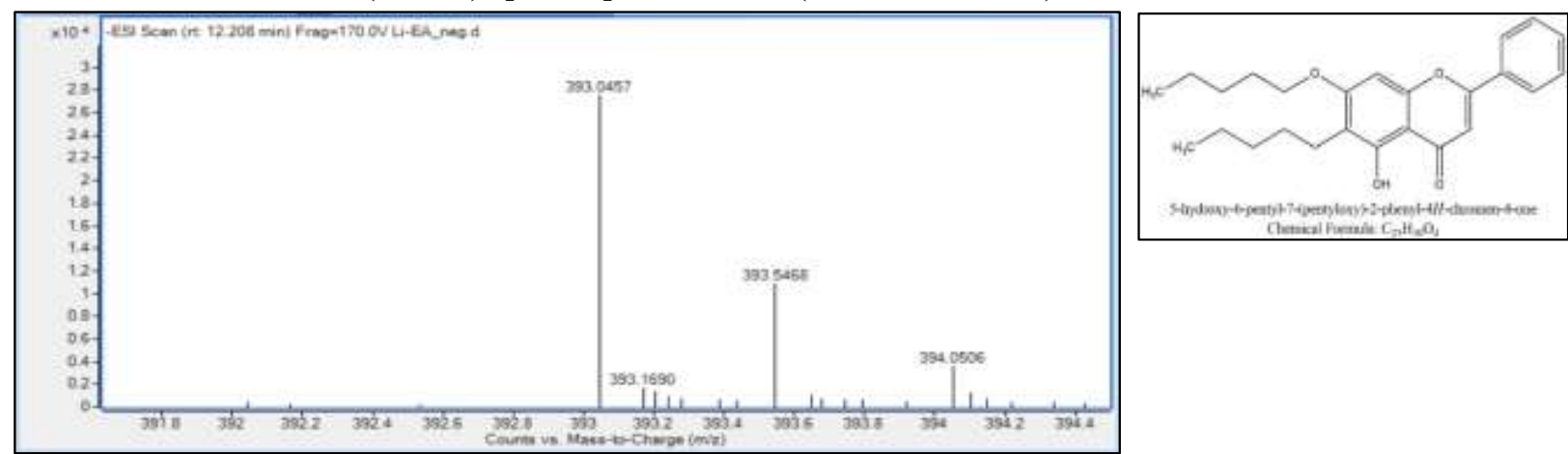

FIGURE 18. Fragmentation pattern from the MS/MS spectrum of the major peak with $\mathrm{m} / \mathrm{z}$ value 393.0457

Peak no. 14, Name: 1,2,3,4,6-penta- $\boldsymbol{O}$-galloly- $\boldsymbol{\beta}$-D-glucose

Exact Mass: 940.1182, (m/z-H); [m/z-1] = 939.1182 (RT: $12.988 \mathrm{~min})$
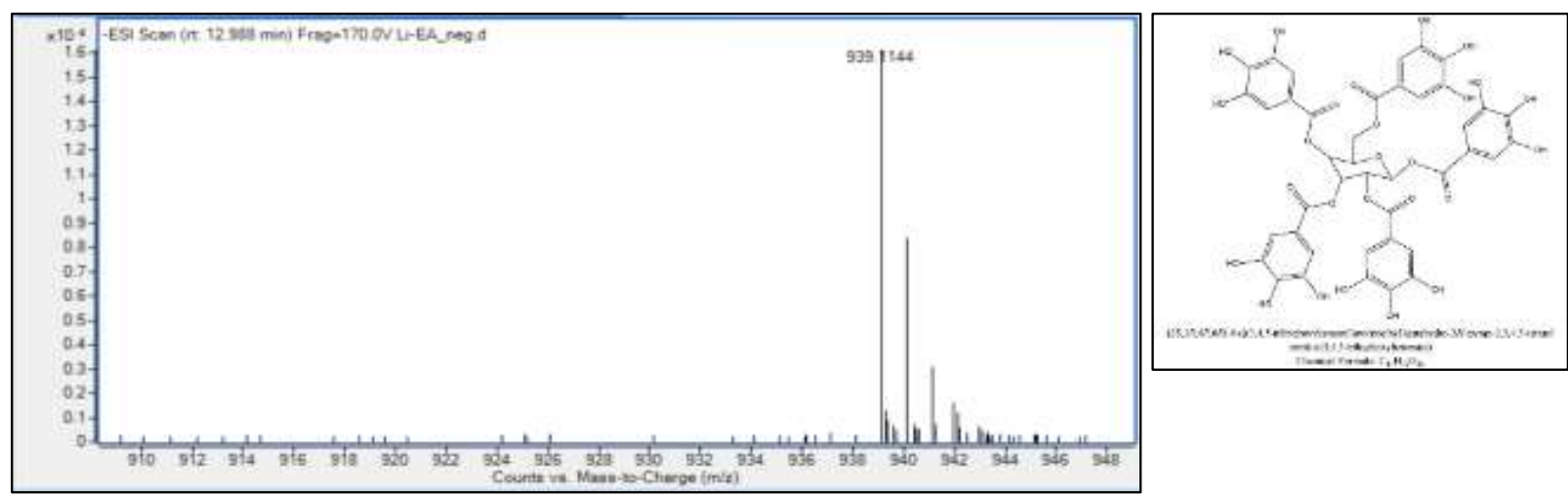

FIGURE 19. Fragmentation pattern from the MS/MS spectrum of the major peak with $\mathrm{m} / \mathrm{z}$ value 939.1144 
Peak no. 15, Name: Luteolin-7-O-glucoside.

Exact Mass: 448.1006, (m/z-H); [m/z-1] $=447.1006$ (RT: $14.366 \mathrm{~min})$
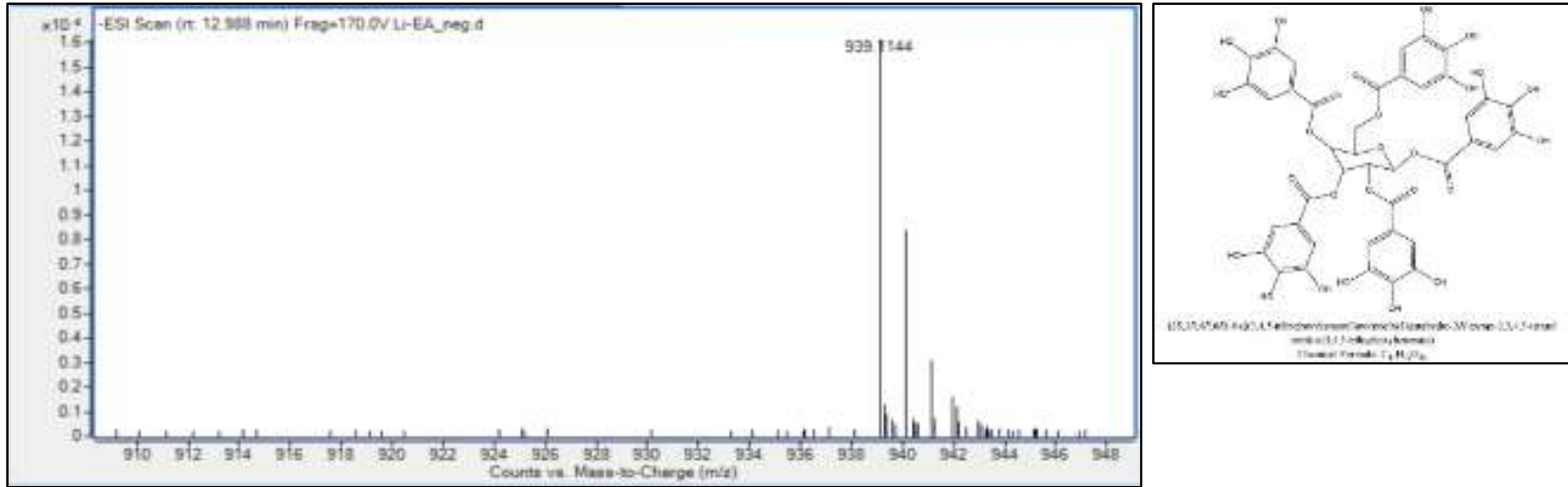

FIGURE 20. Fragmentation pattern from the MS/MS spectrum of the major peak with $\mathrm{m} / \mathrm{z}$ value 447.0929

Peak no. 16, Name: Apiin

Exact Mass: 564.1479, (m/z-H); [m/z-1] = 563.1479 (RT: $14.432 \mathrm{~min})$
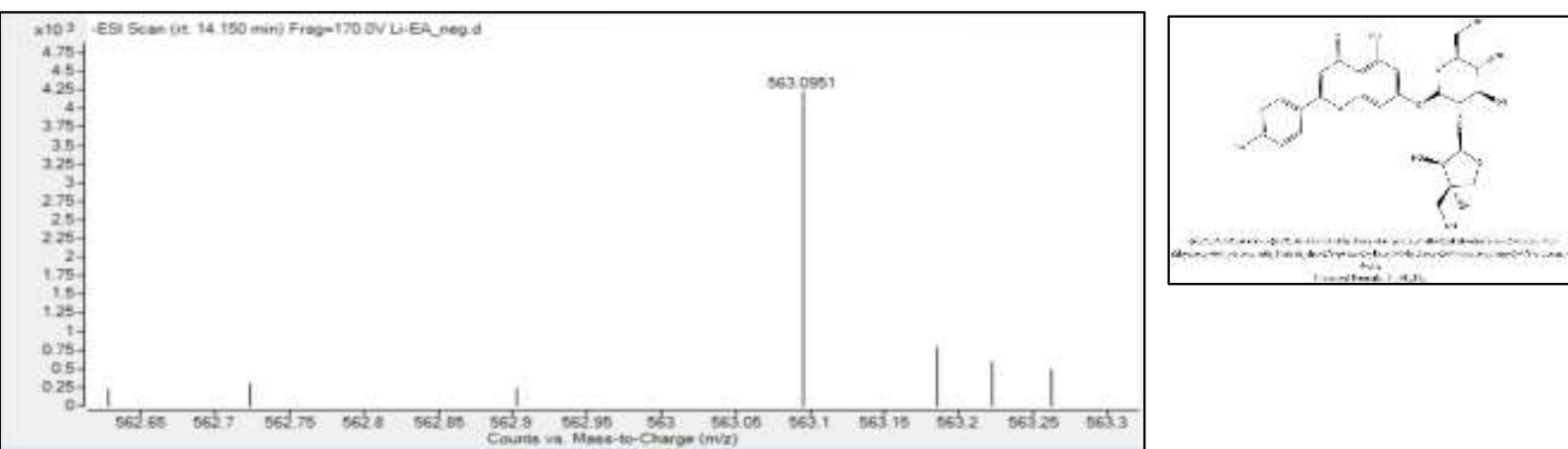

FIGURE 21. Fragmentation pattern from the MS/MS spectrum of the major peak with $\mathrm{m} / \mathrm{z}$ value 563.095

Peak no. 17, Name: Lawsone

Exact Mass:174.0317, [m/z-1] = 173.0317( RT $14.598 \mathrm{~min})$
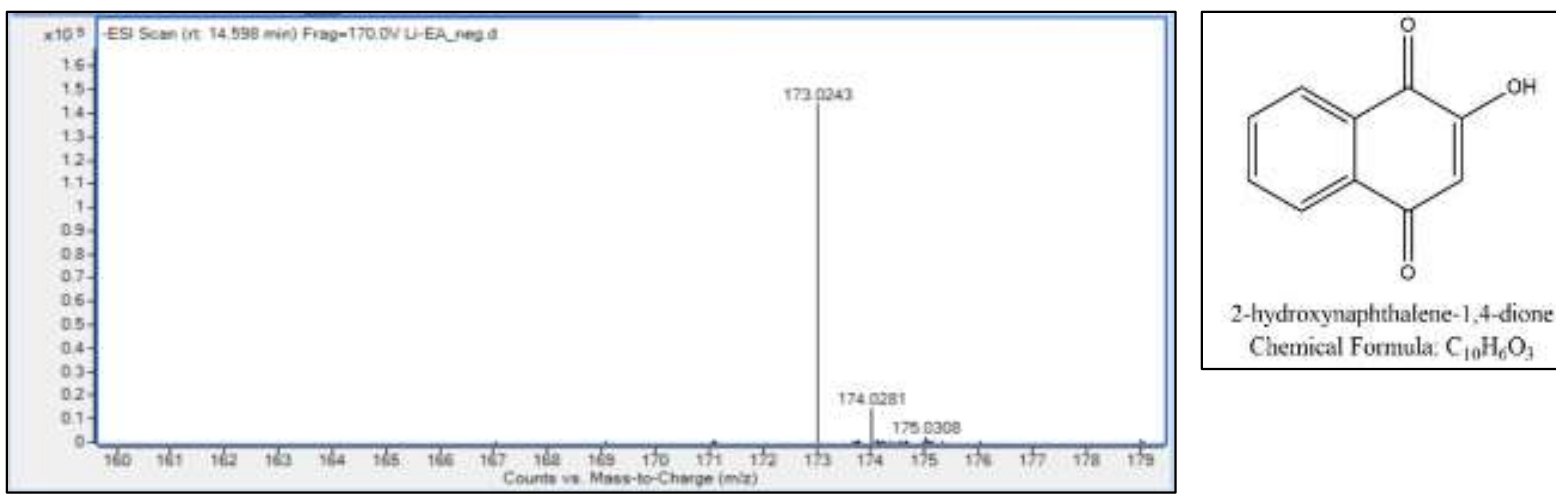

2-hydroxynaphthalene-1 4-dione Chemical Formula. $\mathrm{C}_{10} \mathrm{H}_{6} \mathrm{O}_{3}$

FIGURE 22. Fragmentation pattern from the MS/MS spectrum of the major peak with $\mathrm{m} / \mathrm{z}$ value 173.0242 
Peak no. 18, Name: Isoplumbagin

Exact Mass: 188.0473, (m/z-H); [m/z-1] = 187.0473 (RT: $14.847 \mathrm{~min})$
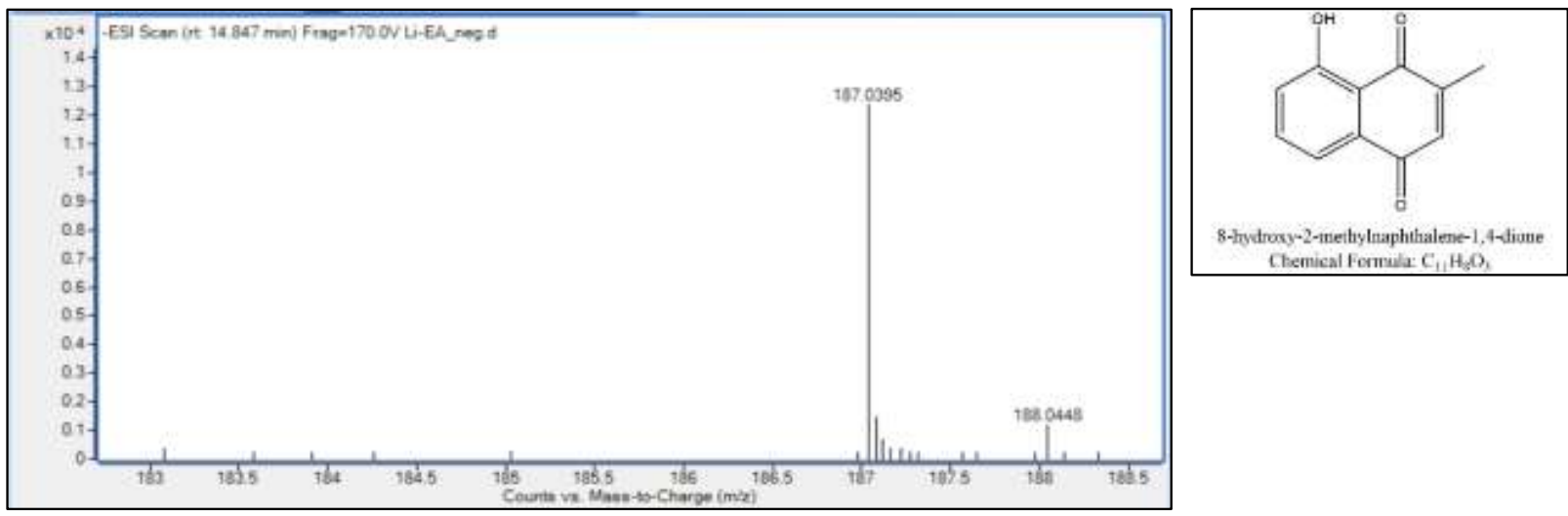

FIGURE 23. Fragmentation pattern from the MS/MS spectrum of the major peak with $\mathrm{m} / \mathrm{z}$ value 187.0395

Peak no. 19, Name: Daphnorin

Exact Mass: 514.1111, (m/z-H); [m/z-1] = 513.1111 (RT: $15.411 \mathrm{~min})$
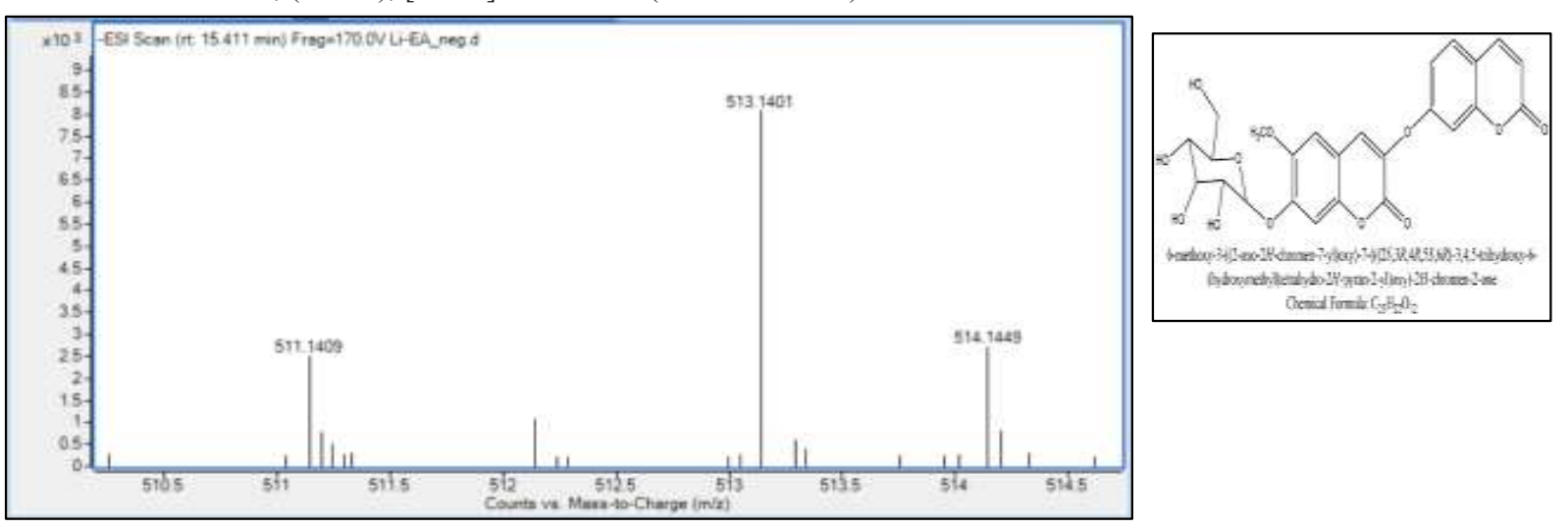

FIGURE 24. Fragmentation pattern from the MS/MS spectrum of the major peak with $\mathrm{m} / \mathrm{z}$ value 513.1401

Peak no. 21, Name: Scutellarin

Exact Mass: 462.0798, (m/z-H); [m/z-1] = 461.0798 (RT: $16.407 \mathrm{~min})$
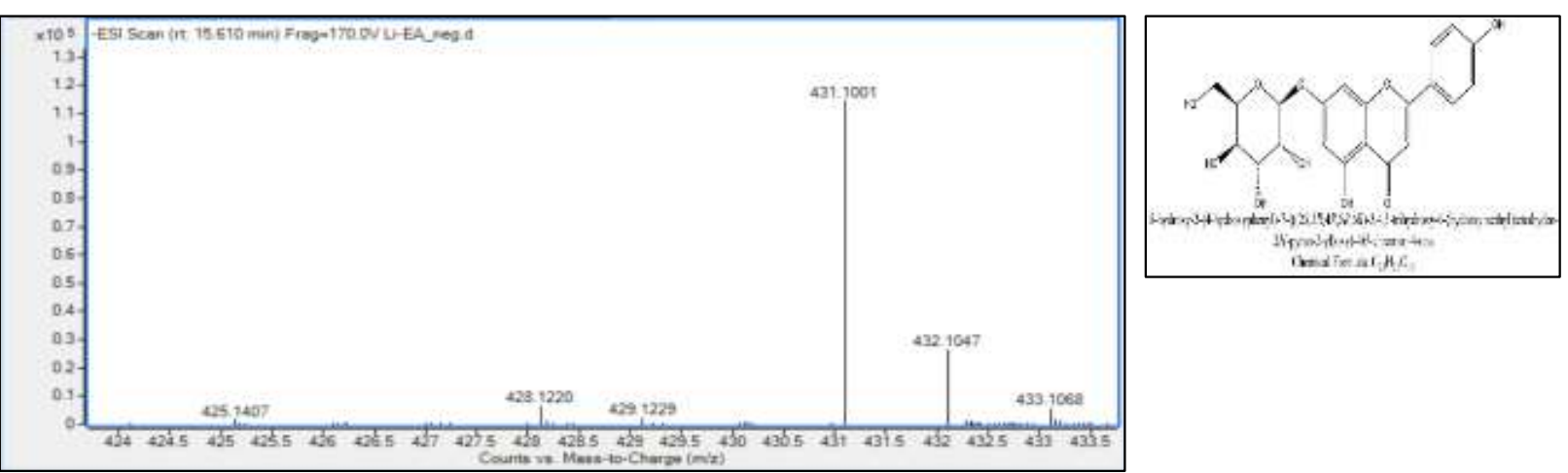

FIGURE 25. Fragmentation pattern from the MS/MS spectrum of the major peak with $\mathrm{m} / \mathrm{z}$ value 413.1001 
Peak no. 21, Name: Scutellarin

Exact Mass: 462.0798, (m/z-H); [m/z-1] = 461.0798 (RT: $16.407 \mathrm{~min})$
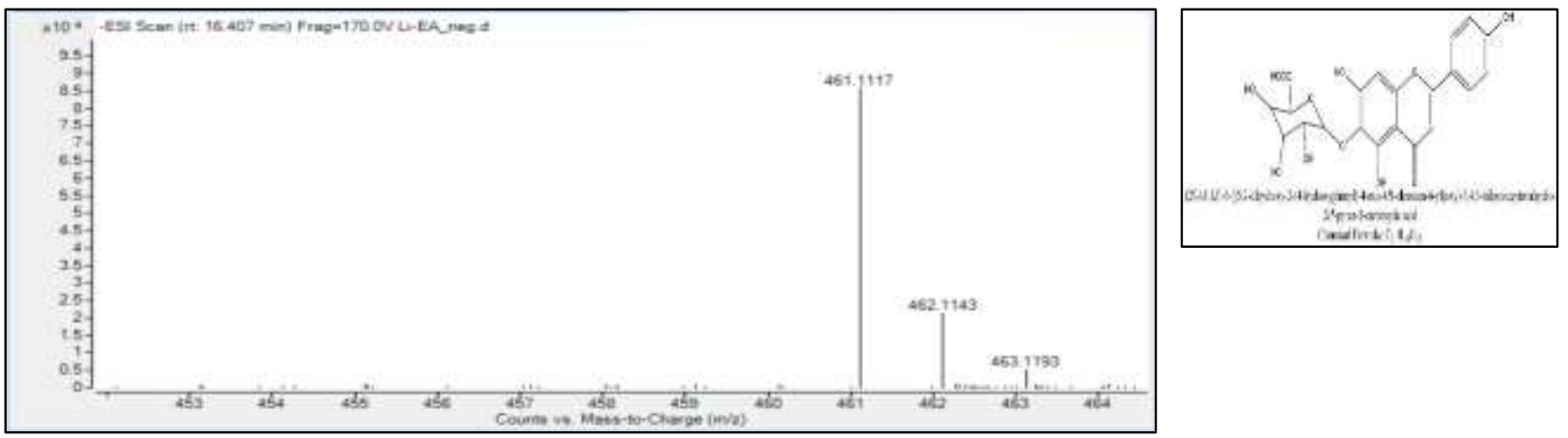

FIGURE 26. Fragmentation pattern from the MS/MS spectrum of the major peak with $\mathrm{m} / \mathrm{z}$ value 461.1117

Peak no. 22, Name: Luteolin

Exact Mass: 286.0477, (m/z-H); [m/z-1] = 285.0477 (RT: $18.631 \mathrm{~min})$
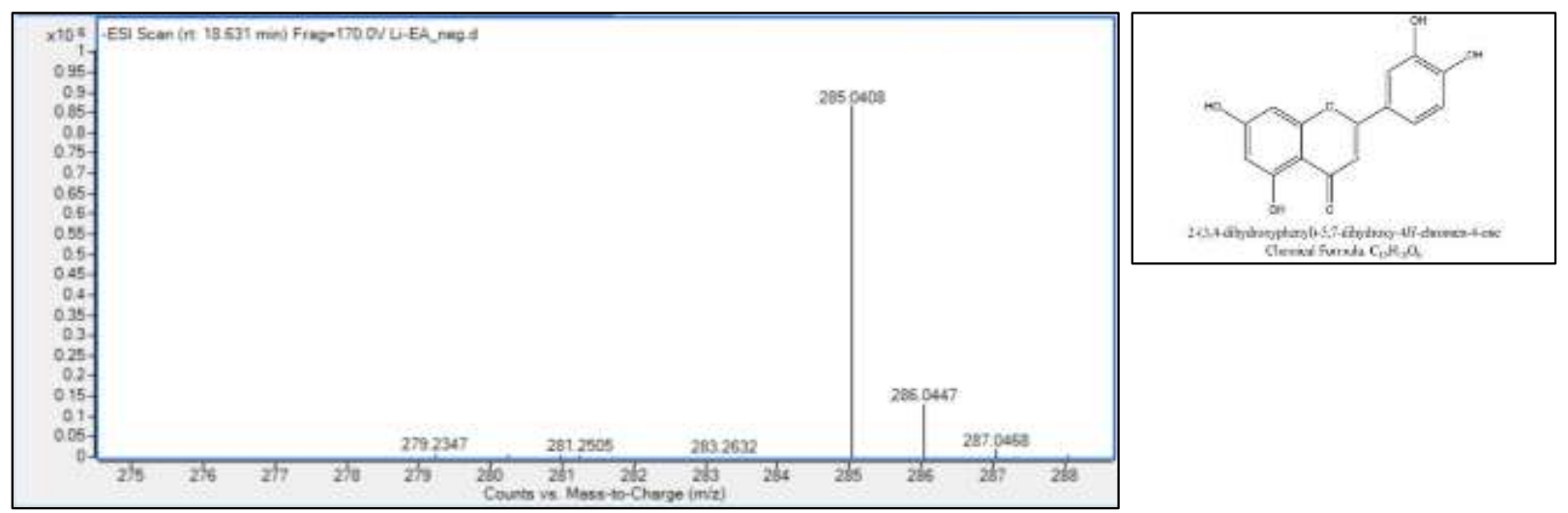

FIGURE 27. Fragmentation pattern from the MS/MS spectrum of the major peak with $\mathrm{m} / \mathrm{z}$ value 285.0408

Peak no. 23, Name: Apigenin

Exact Mass: 270.0528, (m/z-H); [m/z-1] = 269.0528 (RT: 20.025 min)
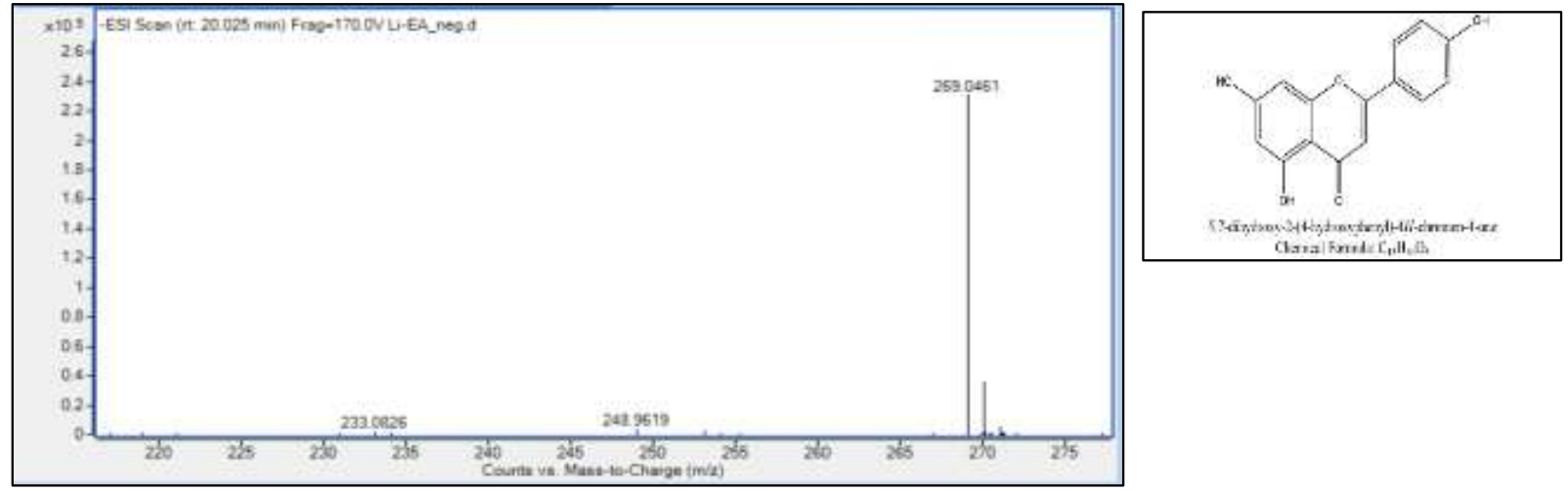

FIGURE 28. Fragmentation pattern from the MS/MS spectrum of the major peak with $\mathrm{m} / \mathrm{z}$ value 269.0461 
Peak no. 24, Name: Daphneside

Exact Mass: 502.1323, (m/z-H); [m/z-1] = 501.1323 (RT: $22.763 \mathrm{~min})$
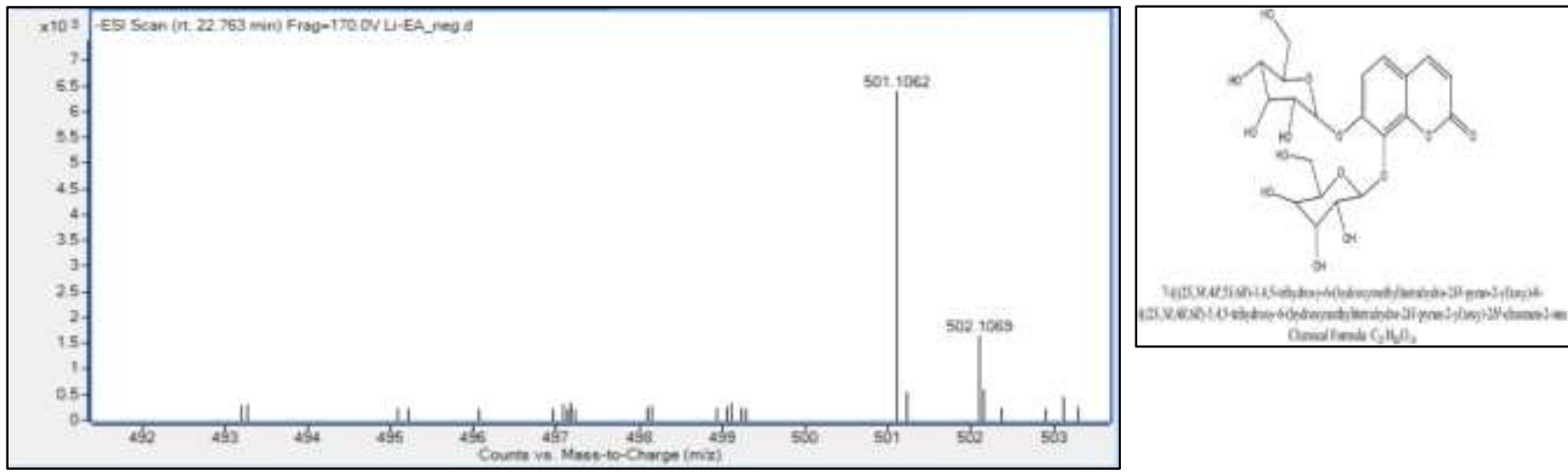

FIGURE 29. Fragmentation pattern from the MS/MS spectrum of the major peak with $\mathrm{m} / \mathrm{z}$ value 501.1062

Peak no. 25, Name: Acacetin

Exact Mass: 284.0685, (m/z-H); [m/z-1] = 283.0685 (RT: $25.004 \mathrm{~min}$ )
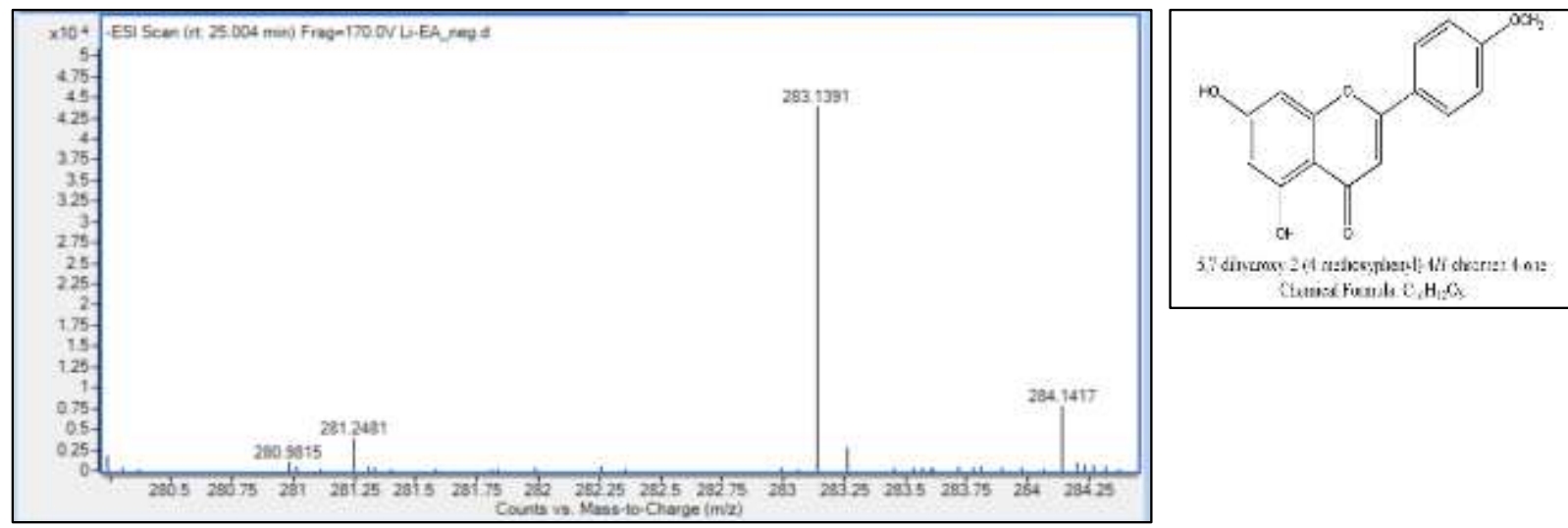

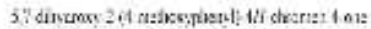

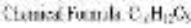

FIGURE 30. Fragmentation pattern from the MS/MS spectrum of the major peak with $\mathrm{m} / \mathrm{z}$ value 283.1391

Peak no. 26, Name: 3',4' dimethoxyflavone

Exact Mass: 298.0841, (m/z-H); [m/z-1] = 297.0841 (RT: $25.651 \mathrm{~min})$
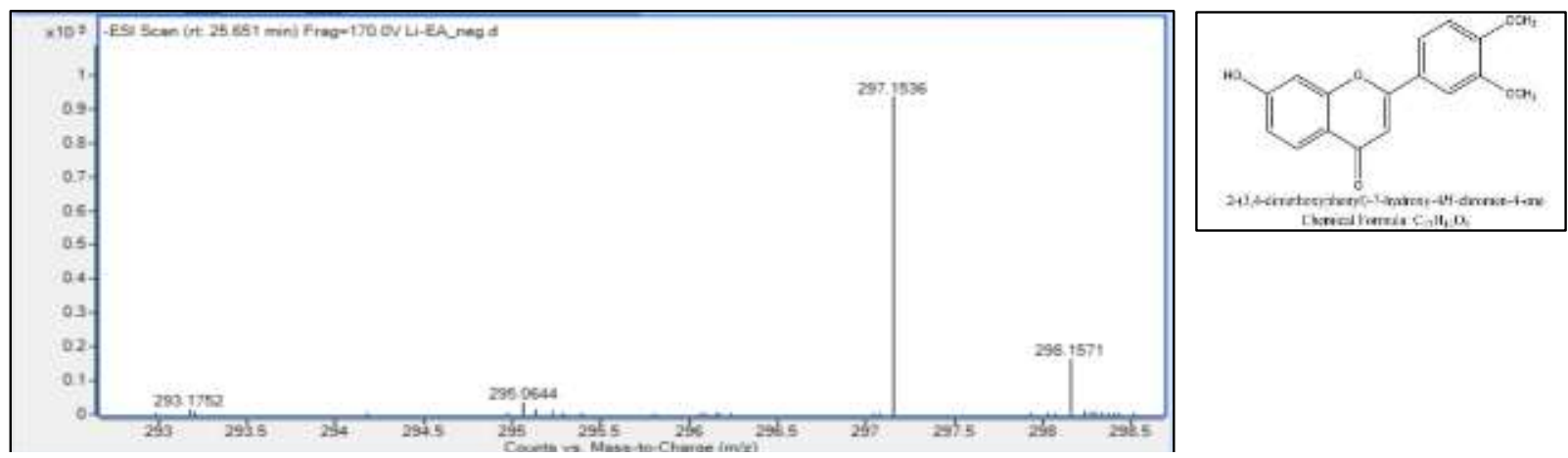

FIGURE 31. Fragmentation pattern from the MS/MS spectrum of the major peak with $\mathrm{m} / \mathrm{z}$ value 297.1536 
Peak no. 27, Name: Lacoumarin

Exact Mass: 218.0579, (m/z-H); [m/z-1] = 217.0579 (RT: $38.198 \mathrm{~min})$
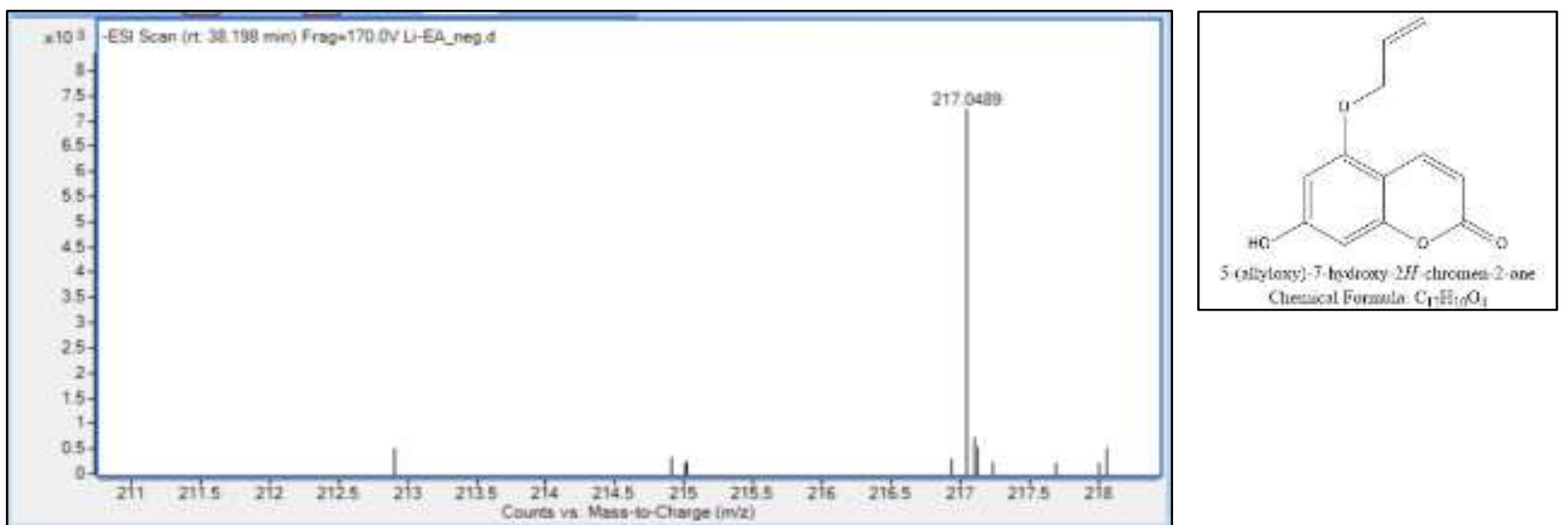

FIGURE 32. Fragmentation pattern from the MS/MS spectrum of the major peak with $\mathrm{m} / \mathrm{z}$ value 217.0489

\section{CONCLUSION}

Many researchers had reported the antioxidant activities of Henna extracts. The antioxidant activities have confirmed the role of the phenolic compounds in the observed activities (Aqil et al. 2006; Hosein \& Zinab 2007; Hsouna et al. 2011; Molina-García et al. 2018).

Compounds such as lalioside (peak no. 11) as a potential source of new natural antioxidants (Hsouna et al. 2011), apiin (peak no. 16), lawsone (peak no. 17), apigenin (peak no. 23), luteolin (peak no. 22), cosmosiin (peak no. 20), p-coumaric acid (peak no. 29), 2-methoxy3-methyl-1,4-naphthoquinone (peak no. 30) produced by the leaves, displayed good antioxidant activities against ABTS [2,2'-azino-bis (3-ethylbenzothiazoline-6sulphonic acid)] compared to ascorbic acid (Mikhaeil et al. 2004) as shown in Table 2.

We manage to detect compound esculetin in peak no. 9 (Figure 14), lalioside (Figure 16), apiin (Figure 21), lawsone (Figure 22), cosmosiin (Figure 25), luteolin (Figure 27), apigenin (Figure 28), in the LC-MS analysis which were recorded by Hsouna et al. (2011) as compound possess good antioxidant activity.

Compound fraxetin is a natural coumarin clinically use as anticoagulant agents shown in peak no. 10 (Figure 15) (Liang et al. 2017). Compound 3',4' dimethoxyflavone in peak no. 12 have shown its potential for anticancer properties (Figure 17) (Lee \& Safe 2000) and compound 3',4'-dimethoxyflavone in peak no. 26 (Figure 31) also have shown anticancer treatment (Kaur et al. 2013). Compound luteolin-7-Oglucoside indicated in peak no. 15 (Figure 20) (Park \& Song 2018) and compound isoplumbagin indicated in peak no. 18 (Figure 23) (Ponugoti 2018) were reported to have the potential for anti-inflammatory activity.
Zhu et al. (2017) have demonstrated in clinical studies of compound scutellarin in peak no. 21 show the potential of an anti-tumour agent for cancer treatment (Figure 26). Compound acacetin indicated in peak no. 25 (Figure 30) is an O-methylated flavone that exhibits anti-inflammatory and antiviral activity which were prevented with HIV-1 activation (Kumar \& Pandey 2013).

From the investigations, an extract of L. inermis have shown predominantly the presence of phenolic compounds such as coumarins, flavonoids, naphthalene, and gallic acid (Figure 8) derivatives which could be glycosylated (Hsouna et al. 2011). Wide range of biological activities have been attributed to henna, including antibacterial, virucidal (inactivate viruses), antiparasitic, anti-inflammatory (Imam et al. 2013), analgesic (relieve pain) and anticancer properties, as well as hepatoprotective, immunomodulatory, anthelminthic, antitrypanosomal (control sleeping sickness) and antioxidant activities (Semwal et al. 2014).

It also provided useful evidence regarding the safety of this traditional medicinal plant as mentioned in the ethnobotany for medicinal bloated and stomach ache in Malay medicine reported in the book medicine in Malay villages vol 2 (Werner 2002). Therefore, this finding is suitable for further research on the gastroprotective activity of $L$. inermis.

\section{ACKNOWLEDGEMENTS}

The authors gratefully acknowledge the financial support from the transdisciplinary research grant scheme (TRGS/1/2014/UM/01/2) sub cluster: Bioassayguided fractionation of selected plants and structural elucidation of isolated pure compounds. We also thank 
phytochemical lab, Department of Chemistry, Faculty Science, University Malaya for the support and guidance.

\section{REFERENCES}

Acquaviva, A., Jones, A., Dennis, G.R., Shalliker, R.A. \& Soliven, A. 2018. Phenolic profiling of complex tea samples via simultaneous multiplexed detection employing reaction flow HPLC columns and colorimetric post-column derivatization. Microchem. J. 138: 533-539.

Agarwal, P., Alok, S. \& Verma, A. 2014. An update on Ayurvedic herb Henna (Lawsonia inermis L.): A review. Int. J. Pharm. Sci. Res. 5(2): 330-339.

Ahmad, I. \& Beg, A.Z. 2001. Antimicrobial and phytochemical studies on 45 Indian medicinal plants against multi-drug resistant human pathogens. J. Ethnopharmacol. 74(2): 113-123.

Alothman, M., Bhat, R. \& Karim, A.A. 2009. Antioxidant capacity and phenolic content of selected tropical fruits from Malaysia, extracted with different solvents. Food Chem. 115(3): 785-788.

Aqil, F., Ahmad, I. \& Mehmood, Z. 2006. Antioxidant and free radical scavenging properties of twelve traditionally used Indian medicinal plants. Turk. J. Biol. 30: 177-183.

Babula, P., Mikelova, R., Potesil, D., Adam, V., Kizek, R., Havel, L. \& Sladky, Z. 2005. Simultaneous determination of 1, 4-naphtoquinone, lawsone, juglone and plumbagin by liquid chromatography with UV detection. Biomed Papers. 149: 25-28.

Brahmachari, G. 2018. Spectroscopic Properties of Natural Flavonoids. World Scientific Publishing Company Pte. Limited.

Chandrakalavathi, T., Sudha, V., Sindhuja, M., Harinipriya, S. \& Jeyalakshmi, R. 2018. Photosonoelectrochemical analysis of Lawsonia inermis (henna) and artificial dye used in the tattoo and dye industry. J. Photochem. Photobiol. A: Chem. 360: 44-57.

Chaudhary, G., Goyal, S. \& Poonia, P. 2010. Lawsonia inermis Linnaeus: A phytopharmacological review. Int. J. Pharm. Sci. Drug Res. 2(2): 91-98.

Ebrahimi, I. \& Parvinzadeh Gashti, M. 2016. Extraction of polyphenolic dyes from henna, pomegranate rind and Pterocarya fraxinifolia for nylon 6 dyeing. Color Technol. 132(2): 162-176.

Gull, I., Sohail, M., Aslam, M.S. \& Amin Athar, M. 2013. Phytochemical, toxicological and antimicrobial evaluation of Lawsonia inermis extracts against clinical isolates of pathogenic bacteria. Ann. Clin. Microb. Anti. 12: 36.

Hosein, H.K.M. \& Zinab, D. 2007. Phenolic compounds and antioxidant activity of henna leaves extracts (Lawsonia inermis). World J. of Dairy Food Sci. 2(1): 38-41.

Hsouna, A.B., Trigui, M., Culioli, G., Blache, Y. \& Jaoua, S. 2011. Antioxidant constituents from Lawsonia inermis leaves: Isolation, structure elucidation and antioxidative capacity. Food Chem. 125(1): 193-200.

Imam, H., Mahbub, N.U., Khan, M.F., Hana, H.K. \& Sarker, M.M.R. 2013. Alpha-amylase enzyme inhibitory and antiinflammatory effect of Lawsonia inermis. Pak. J. Bio. Sci. 16(23): 1796-1800.

Jabborova, D., Davranov, K. \& Egamberdieva, D. 2019. Antibacterial, antifungal, and antiviral properties of medical plants. In Medically Important Plant Biomes: Source of Secondary Metabolites. Springer, Singapore. pp. 51-65.

Kahkeshani, N., Farzaei, F., Fotouhi, M., Alavi, S.S., Bahramsoltani, R., Naseri, R., Momtaz, S., Abbasabadi, Z., Rahimi, R., Farzaei, M.H. \& Bishayee, A. 2019. Pharmacological effects of gallic acid in health and disease: A mechanistic review. Iran J. Basic Medic. Sci. 22(3): 225-237.

Kaur, K., Mirlashari, M.R., Kvalheim, G. \& Kjeldsen-Kragh, J. 2013. 3', 4'-Dimethoxyflavone and valproic acid promote the proliferation of human hematopoietic stem cells. Stem Cell Res. Ther. 4(3): 1-8.

Kumar, S. \& Pandey, A.K. 2013. Chemistry and biological activities of flavonoids: An overview. Sci. World J. 2013: Article ID. 162750.

Molina-García, L., Martínez-Expósito, R., Fernández-de Córdova, M.L. \& Llorent-Martínez E.J. 2018. Determination of the phenolic profile and antioxidant activity of leaves and fruits of Spanish Quercus coccifera. J. Chem. 2018: 1-9.

Lee, J.E. \& Safe, S. 2000. 3', 4'-Dimethoxyflavone as an aryl hydrocarbon receptor antagonist in human breast cancer cells. Tox. Sci. 58(2): 235-242.

Liang, C., Ju, W., Pei, S., Tang, Y. \& Xiao, Y. 2017. Pharmacological activities and synthesis of esculetin and its derivatives: A mini-review. Mol. 22(3): 387.

Mikhaeil, B.R., Badria, F.A., Maatooq, G.T. \& Amer, M.M. 2004. Antioxidant and immunomodulatory constituents of henna leaves. Z. Naturforschung C. 59(7-8): 468-476.

Muhammad, H.S. \& Muhammad, S. 2005. The use of Lawsonia inermis Linn (Henna) in the management of burn wound infection. African J. Biotechnol. 4: 934-937.

Oroian, M. \& Escriche, I. 2015. Antioxidants: Characterization, natural sources, extraction and analysis. Food Res. Int. 74: 10-36.

Othman, M.R., Ahmad, K., Othman, R., Awang, K. \& Yusoff, M.Z. 2020. Use of henna in the Malay world: From traditional uses to scientific approches. In Tibb Nabawi on NatureBased Products, edited by Mohd Yakub@ Zulkifli Mohd Yusoff, Khadher Ahmed \& Rozana Othman. Kuala Lumpur: University of Malaya Press. pp. 99-114.

Park, C.M. \& Song, Y.S. 2018. Comparative analysis of the anti-inflammatory activity of luteolin and luteolin-7-Oglucoside against galactosamine/lipopolysaccharide induced hepatotoxicity in mice. Proceed Nutr. Soc. 2018: 77(OCE4).

Pasandi Pour, A. \& Farahbakhsh, H. 2019. Lawsonia inermis L. leaves aqueous extract as a natural antioxidant and antibacterial product. Nat. Pro. Res. 2019: 1-5.

Ponugoti, M. 2018. A pharmacological and toxicological review of Lawsonia inermis. Inter. J. Pharm. Sci. Res. 9(3): 902-915.

Saeed, S.M.G., Sayeed, S.A., Ashraf, S., Naz, S., Siddiqi, R., Ali, R. \& Mesaik, M.A. 2013. A new method for the isolation and purification of lawsone from Lawsonia inermis and its ROS inhibitory activity. Pakistan J. Bot. 45(4): 1431-1436.

Semwal, R.B., Semwal, D.K., Combrinck, S., CartwrightJones, C. \& Viljoen, A. 2014. Lawsonia inermis L. (henna): Ethnobotanical, phytochemical and pharmacological aspects. J. Ethnopharm.155(1): 80-103.

Sharma, R.K., Goel, A. \& Bhatia, A.K. 2016. Lawsonia inermis Linn: A plant with cosmetic and medical benefits. Int. J. App. Sci. Biotechnol. 4(1): 15-20. 
Tan, M.C., Tan, C.P. \& Ho, C.W. 2013. Effects of extraction solvent system, time and temperature on total phenolic content of henna (Lawsonia inermis) stems. Int. Food Res. J. 20(6): 3117-3123

Uddin, N., Siddiqui, B.S. \& Begum, S. 2013. Chemical constituents and bioactivities of Lawsonia alba Lam. (Henna). J. Chem. Soc. Pakistan. 35(2): 476-485.

Werner, R. 2002. Medicines in Malay Villages. Vol 2. Kuala Lumpur: University of Malaya Press.

Xiang, Y.F., Ju, H.Q., Li, S., Zhang, Y.J., Yang, C.R. \& Wang, Y.F. 2010. Effects of 1, 2, 4, 6-tetra-O-galloyl- $\beta$-D-glucose from P. emblica on HBsAg and HBeAg secretion in HepG2. 2.15 cell culture. Virol. Sin. 25(5): 375-380.

Zhu, P.T., Mao, M., Liu, Z.G., Tao, L. \& Yan, B.C. 2017. Scutellarin suppresses human colorectal cancer metastasis and angiogenesis by targeting ephrinb2. American J. Trans. Res. 9(11): 5094-5104.

Muhammad Remy Othman*, Khadher Ahmad, Munirah Abd Razzak \& Zulkifli Mohd Yusoff

Department of Al-Quran and Al-Hadith

Academy of Islamic Studies

University of Malaya

50603 Kuala Lumpur, Federal Territory

Malaysia
Muhammad Remy Othman, Hazrina Hazni \& Khalijah Awang Department of Chemistry

Faculty of Science

University of Malaya

50603 Kuala Lumpur, Federal Territory

Malaysia

Rozana Othman \& Abdul Aziz Ismail

Department of Pharmaceutical Chemistry

Faculty of Pharmacy

University of Malaya

50603 Kuala Lumpur, Federal Territory

Malaysia

Rozana Othman, Hazrina Hazni \& Khalijah Awang

Centre for Natural Products Research and Drug Discovery (CENAR)

University of Malaya

50603 Kuala Lumpur, Federal Territory

Malaysia

*Corresponding author; email: remy_um86@siswa.um.edu.my

Received: 25 October 2019

Accepted: 7 March 2020 